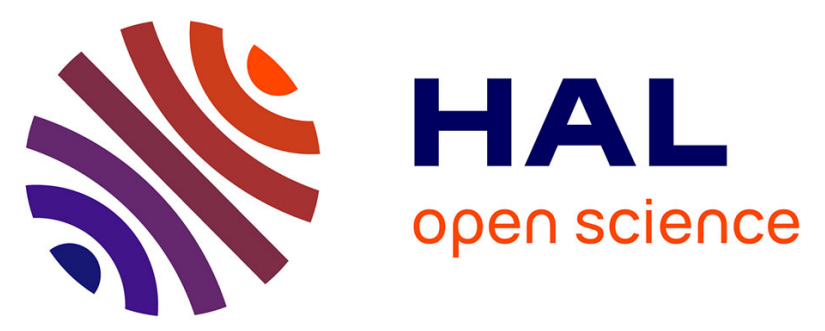

\title{
Manipulating the physical states of confined ibuprofen in SBA-15 based drug delivery systems obtained by solid-state loading: Impact of the loading degree
}

\author{
B. Malfait, Natália T. Correia, Carmen Ciotonea, Jérémy Dhainaut, \\ Jean-Philippe Dacquin, Sébastien Royer, Nicolas Tabary, Yannick Guinet, \\ Alain Hedoux
}

\section{To cite this version:}

B. Malfait, Natália T. Correia, Carmen Ciotonea, Jérémy Dhainaut, Jean-Philippe Dacquin, et al.. Manipulating the physical states of confined ibuprofen in SBA-15 based drug delivery systems obtained by solid-state loading: Impact of the loading degree. Journal of Chemical Physics, 2020, 153 (15), pp.154506. 10.1063/5.0020992 . hal-02974158v2

\section{HAL Id: hal-02974158 \\ https://hal.univ-lille.fr/hal-02974158v2}

Submitted on 12 Feb 2021

HAL is a multi-disciplinary open access archive for the deposit and dissemination of scientific research documents, whether they are published or not. The documents may come from teaching and research institutions in France or abroad, or from public or private research centers.
L'archive ouverte pluridisciplinaire HAL, est destinée au dépôt et à la diffusion de documents scientifiques de niveau recherche, publiés ou non, émanant des établissements d'enseignement et de recherche français ou étrangers, des laboratoires publics ou privés. 
Manipulating the physical states of confined ibuprofen in SBA-15 based drug delivery systems obtained by solid-state loading: impact of the loading degree

\author{
Benjamin Malfait ${ }^{a, b}$, Natália T. Correia ${ }^{a}$, Carmen Ciotonea ${ }^{c}$, Jérémy Dhainaut ${ }^{c}$, Jean- \\ Philippe Dacquinc, Sébastien Royer ${ }^{c}$, Nicolas Tabary ${ }^{a}$, Yannick Guinet ${ }^{a}$, Alain \\ Hédoux $\mathrm{a}^{*}$
}

aUniv. Lille, CNRS, INRAE, Centrale Lille, UMR 8207 - UMET - Unité Matériaux et Transformations, F-59000 Lille, France

bInstitute of Physics of Rennes, CNRS-University of Rennes 1, UMR 6251, F35042 Rennes, France

CUniv. Lille, CNRS, Centrale Lille, ENSCL, Univ. Artois, UMR 8181 - UCCS Unité de Catalyse et Chimie du Solide, F-59000 Lille, France

*corresponding author : alain.hedoux@univ-lille.fr

\begin{abstract}
Using the Milling-Assisted Loading (MAL) solid-state method, for loading a poorly water-soluble drug (ibuprofen, IBP) within SBA-15 matrix has given the opportunity to manipulate the physical state of drugs for optimizing bioavailability. MAL method makes it easy to control and analyze the influence of the degree of loading on the physical state of IBP inside SBA-15 matrix with an average pore diameter of $9.4 \mathrm{~nm}$. It was found that the density of IBP molecules in an average pore size has a direct influence both on the glass transition and the mechanism of crystallization. Detailed analyzes of the crystallite distribution and melting by Raman
\end{abstract}


mapping, X-Ray diffraction and differential scanning calorimetry, have shown that the crystals are localized in the core of the channel and surrounded by a liquid monolayer. The results of these complementary investigations have been used for determining the relevant parameters (related to the SBA-15 matrix and to IBP molecule) for determining the nature of the physical state of the confined matter.

Keywords: Confinement - physical state - bioavailability - loading degree Low-wavenumber Raman mapping - sustained release 


\section{Introduction}

In the pharmaceutical area, most of active molecules are synthesized in the solid state and more specifically in a crystalline poorly water-soluble form ${ }^{1}$. Despite the thermodynamic stability of the crystalline state, its low bioavailability is a major obstacle to the development of new drugs. Different strategies are been developed to tackle this problem. Some are based on the use of the amorphous form, or metastable polymorphs, with potentially higher apparent solubility but thermodynamically very unstable relatively to crystalline state. This overtime instability is a consequent drawback for pharmaceutical applications and therefore requires compromising solutions ${ }^{2}$

Mesoporous silica (MPS) matrices are recognized as promising drug delivery systems $^{3}$ due to their high surface area, tunable pore diameter and morphology, easily modifiable chemical surface ${ }^{3-5}$. In addition, the inorganic nature of MPS provides a protective shield against physiological attacks ${ }^{3}$. Confinement of drug molecules into MPS matrices allows stabilizing highly soluble physical states as metastable polymorphs $^{6}$ or amorphous ${ }^{7}$ forms of poorly water-soluble crystalline drugs.

In a more general context, it is well known that confinement is a pathway to manipulate the physical state by varying the pore diameter ${ }^{8,9}$. The amorphous state can be stabilized when the pore diameter is small enough to inhibit the crystallization, i.e. the nucleation process being inhibited by spatial restriction ${ }^{8-11}$. The attempt to establish an empirical correlation between the diameter of the pores and the size of the molecule and its physical state or states under confinement can be found in the literature. From the point of view of the pharmaceutical industry, the search for empirical correlations can be interesting for the rational use of drugs inside the MPS aiming to improving the solubilization of the drug by amorphization and the stabilization 
of the drug by confinement. Few examples of such results on low molecular weight compounds, including non-pharmaceuticals, can be found in the literature. SliwinskaBartkowiak et al. ${ }^{12}$ reported experimental measurements on nitrobenzene into controlled pore glass (CPG), Vycor, and MCM-41 with pore diameter ranging from 2.8 to $50 \mathrm{~nm}$, and molecular simulations on tetrachloromethane adsorbed in straight cylindrical pores of different pore diameters. Their results have shown that homogeneous crystallization was observed for average pore diameter larger than $20 \sigma$, where $\sigma$ is the molecular diameter. In pore diameters between $12 \sigma$ and $20 \sigma$, the confined phase was found to be inhomogeneous, with a frustrated crystalline structure coexisting with amorphous regions. While no crystallization was observed for pores with diameters lower than $12 \sigma$. A similar threshold was also observed for other materials confined within silica mesopores. In the case of benzene ${ }^{13}$ confined into SBA-15 and MCM-41, with pore diameter ranging from 8.7 to $2.4 \mathrm{~nm}$, crystallization was detected only in the pores of $8.7 \mathrm{~nm}$ whereas no crystallization was detected in $4.7 \mathrm{~nm}$ (or $<4.7 \mathrm{~nm}$ ) which corresponds to roughly $10 \sigma$. For water ${ }^{14}$ confined into MCM41 , crystallization was observed into the $3 \mathrm{~nm}$ pore diameter while it did not occurred for $2.5 \mathrm{~nm}$ pore diameter. The water molecule dimension ${ }^{15}$ being $0.28 \mathrm{~nm}$, pore diameters of $3 \mathrm{~nm}$ corresponding to about $11 \sigma$. Very recently, Cheng and McKenna ${ }^{11}$ showed that for a pharmaceutical drug, nifedipine under confinement into CPG ranging from 198 to $7.5 \mathrm{~nm}$, no crystallization was observed in pores ranging from 7.5 and 12 $\mathrm{nm}$ of diameter. The latter pore diameter roughly corresponds to $12 \sigma$. Thus, an empirical threshold of 10-12 molecular diameters appears as the higher limit for pore diameters allowing to confine matter in a completely amorphous state, at least inside the mentioned type of MPS matrices, namely MCM-41, SBA-15 and CPG. It is worth noting that many of the mentioned experimental and theoretical works have suggested 
the presence of a very stable amorphous layer of molecules in interaction with silica walls, surrounding the crystalline matter (see also ref. ${ }^{16-19}$ ). This amorphous layer was indeed observed for several systems like benzene ${ }^{13}$, water ${ }^{19}$, nifedipine ${ }^{11}$, acetaminophen ${ }^{18}$ or ibuprofen ${ }^{20}$, to name a few.

However, there is clearly a lack of systematic information about the loading/filling degree influence on the physical states of encapsulated systems. In a recent paper, Nartowski et al. ${ }^{21}$ performed detailed ${ }^{19} \mathrm{~F}$ solid-state NMR investigations of flufenamic acid (FFA) in mesoporous silica matrices with different pore diameters (3.2 to $29 \mathrm{~nm}$ ) and a large range of loading degrees. For fulfilled pores (loading up to 30 wt.\%), FFA was found as a disordered amorphous phase along with a liquid-like surface layer, in the $3.2 \mathrm{~nm}(\mathrm{MCM}-41)$ and $7.1 \mathrm{~nm}(\mathrm{SBA}-15)$ pore diameters $(<10 \sigma)$. Whereas for the $29 \mathrm{~nm}$ diameter ( > 10 $\sigma$ ) mesostructured cellular foam (MCF) pores, the physical state of FFA showed to be loading degree dependent. At low loading degrees up to 20 wt.\% FFA was completely amorphous. Then, progressively a signal attributed to FFA form I was detected at higher loadings. Moreover, it was shown that only a highly mobile molecular monolayer at the silica surface is present at $15 \mathrm{wt} \%$ loading, while for $20 \mathrm{wt} \%$ and higher, crystalline matter in the core of the pores co-exist with the FFA molecules of the surface-layer, which is almost unaffected by the crystallization.

Several studies in the literature have shown that racemic ibuprofen, the target poorly water-soluble model drug of the present work, is in an amorphous stable state confined in small pore diameters like MCM-41 (Brás et al. ${ }^{22}$, pores diameter $3.6 \mathrm{~nm}$ ), and in SBA-15 with pore diameters of $8.4 \mathrm{~nm}$ (Mellaerts et al. ${ }^{23}$ ), of $8.6 / 8.8 \mathrm{~nm}$ (Izquierdo-Barba et al..$^{24}$ and Brás et al. ${ }^{25}$ ) and of $6 \mathrm{~nm}$ (Malfait et al. $\left.{ }^{26}\right)$. The smallest pores where crystallization of ibuprofen was reported in the literature (Riikonen et al. ${ }^{20}$ ) 
are mesoporous silicon with an average pore diameter of $11.3 \mathrm{~nm}$, corresponding to approximatively $11 \sigma$. Nevertheless, discussion is not easy since different methods of encapsulation, loading degrees and even the method to report the pore diameter (sometimes using BJH adsorption ${ }^{24,25}$ others the BJH desorption ${ }^{20,26}$ branch) are reported in the literature.

In the present study, we propose to investigate the loading degree influence on the physical state and transformation of racemic ibuprofen when supported in a SBA15 matrix with an average pore diameter of $9 \mathrm{~nm}$ (BJH desorption branch or $11 \mathrm{~nm}$ from BJH adsorption branch); close to the $10 \sigma$ threshold. Milling-Assisted Loading (MAL) method was used which allows to precisely control the loading degree of the guest material ${ }^{26}$. This MAL method also overcomes the major drawbacks of the two main existing loading methods for technological use of MPS as drug carriers. Namely, the impregnation method ${ }^{27}$ which requires the use of toxic organic solvents for placing poorly water-soluble drugs in the liquid state and thus, is not consistent with a pharmaceutical application. On the other hand, adsorption of drug molecules into the pores after melting ${ }^{23}$ can induce chemical degradation if the melting temperature is high $^{28}$.

Several experimental techniques, thermogravimetry and differential scanning calorimetry analysis, X-ray powder diffraction and $\mu$-Raman spectroscopy, were used complementarily in order to establish a picture as detailed as possible of the physical states that can co-exist within nanochannels of a SBA-15 matrix of $9 \mathrm{~nm}$ diameter. Additionally, the influence of the physical state on the drug release kinetics was also analyzed, to show the capabilities of modifying the drug release profile only by small changes in the physical state of the confined material. 


\section{Materials and methods}

\subsection{Materials, mesopores synthesis and drug loading procedure}

Racemic ibuprofen (designated herein as IBP) was purchased from Sigma (CAS number 15687-27-1, purity $\geq 99.8 \%$ GC assay) and it was used without further purification.

SBA-15 was used as mesoporous silica matrix. SBA-15 support was synthesized according to the procedure proposed by Zhao et $\mathrm{al}^{29}$. In a typical procedure, $4.00 \mathrm{~g}$ of Pluronic P123 (Aldrich, $\mathrm{PEO}_{20} \mathrm{PPO}_{70} \mathrm{PEO}_{20}, \mathrm{MW}=5800 \mathrm{~g} \cdot \mathrm{mol}^{-1}$, CAS number 9003116$)$ is added to $150 \mathrm{~mL}$ of $\mathrm{HCl}$ solution $(1.6 \mathrm{M})$, and the solution was stirred at $40{ }^{\circ} \mathrm{C}$ for $6 \mathrm{~h}$. Then the silica source, $8.5 \mathrm{~g}$ of TEOS (Sigma-Aldrich, reagent grade $98 \%$, CAS number 78104 ) was added dropwise to the solution and the stirring was maintained for $24 \mathrm{~h}$. The resulting white suspension was submitted to a hydrothermal treatment at $140{ }^{\circ} \mathrm{C}$ for $48 \mathrm{~h}$. This $140{ }^{\circ} \mathrm{C}$ treatment is not common (usually $100^{\circ} \mathrm{C}$ ) and allows to obtain larger pores diameter. The solid was thereafter collected by filtration, washed with distilled water, and dried at $80{ }^{\circ} \mathrm{C}$ for $12 \mathrm{~h}$. The porous SBA-15 support was obtained by calcination at $550^{\circ} \mathrm{C}$ for $6 \mathrm{~h}$ in a muffle oven (the temperature was raised at a rate of $1.5^{\circ} \mathrm{C} / \mathrm{min}$ ). The as-synthesized SBA-15 powder was handled in a desiccator.

SBA-15 matrix is characterized by a uniform and narrow pore diameter distribution (Figure S1) determined by Barrett-Joyner-Halenda (BJH) method ${ }^{30}$ on the desorption branch (average pore diameter $D_{p, \text { des }}=9.4 \mathrm{~nm}$ ) and on the adsorption branch (average pore diameter $D_{p, a d s}=11.4 \mathrm{~nm}$ ) from nitrogen adsorption/desorption experiments at $-196.15^{\circ} \mathrm{C}$. The specific surface area was evaluated $\left(\mathrm{S}_{\mathrm{BET}}=457 \mathrm{~m}^{2} / \mathrm{g}\right)$ from the linear portion of Brunauer-Emmett-Teller (BET) method plots ${ }^{31}$. The total pore 
volume $\left(V_{p}=1.16 \mathrm{~cm}^{3} / \mathrm{g}\right)$ was calculated from the volume adsorbed at $\mathrm{P} / \mathrm{P}_{0}=0.99$; the micropores volume $\left(V_{\mu}=0.05 \mathrm{~cm}^{3} / \mathrm{g}\right)$ was evaluated using the t-plot $\left(\mathrm{P} / \mathrm{P}_{0}=0.2-0.5\right)$. Hereafter this SBA-15 matrix will be designated as SBA-15[9nm].

Ibuprofen loading was carried out using the Milling-Assisted Loading (MAL) method $^{26}$. Co-milling of IBP and SBA-15 solid mixtures was systematically performed at room temperature, using a Retsch Mixer Mill MM400 (Haan, Germany). According to this method $^{26}$, the as-received crystalline IBP and MPS powders were sealed in an agate jar $(5 \mathrm{~mL})$ with one agate ball $(\varnothing=7 \mathrm{~mm})$. The jar, filled to a third of its volume, was placed on the mixer arm, which vibrates at a frequency of $30 \mathrm{~Hz}$. To avoid any overheating of IBP, milling periods ( $5 \mathrm{~min}$ ) were alternated with pause periods ( $2 \mathrm{~min})$. Several composites were prepared corresponding to co-milling of as-received Ibuprofen with SBA-15 matrix in an IBP weight \% of 10, 20, 25, 35 and 40. The composites are hereinafter identified by as IBPX\%:SBA-15, where $\mathrm{X}$ is the weight \% of IBP.

\subsection{Methods}

Thermogravimetry analyses (TGA) of the as-synthetized SBA-15[9nm] matrix, bulk ibuprofen and IBP:SBA-15 composites were performed with a Thermogravimetric Analyzer Q500 (TA Instruments, Guyancourt, France) operating under a highly pure nitrogen gas at a flow rate of $60 \mathrm{~mL} / \mathrm{min}$. Samples of around $1-3 \mathrm{mg}$ were heated from room temperature to $550{ }^{\circ} \mathrm{C}$ at a heating rate of $5{ }^{\circ} \mathrm{C} / \mathrm{min}$. The silica mesoporous matrices are thermally stable at temperatures higher than $550^{\circ} \mathrm{C}$. TGA curve (not shown) of the unloaded SBA-15[9nm] matrix (handled in a desiccator) presents only a weight loss of around 0.6 wt.\% below $100{ }^{\circ} \mathrm{C}$ attributed to the removal of weakly physically adsorbed water. 
Differential Scanning Calorimetry (DSC) experiments were carried with $i$ ) a DSC Q1000 equipped with a Refrigerated Cooling System, in the temperature range from $80^{\circ} \mathrm{C}$ to $100^{\circ} \mathrm{C}$, at heating rate of $1^{\circ} \mathrm{C} / \mathrm{min}$, and ii) a DSC Q10 equipped with a Liquid Nitrogen Cooling System, between $-150{ }^{\circ} \mathrm{C}$ and $100^{\circ} \mathrm{C}$, at heating rate of $20^{\circ} \mathrm{C} / \mathrm{min}$. The former procedure allows to probe the stability against crystallization while the latter was chosen in order to maximize the calorimetric signal and highlighting the glass transition event in the IBP:SBA-15 composites. TA Instruments (Guyancourt, France) provided both devices. Samples of 3-8 $\mathrm{mg}$ were encapsulated in Tzero (aluminum) hermetic pans with Tzero (aluminum) hermetic lids. During all measurements, the calorimeter head of the DSC Q1000 was flushed with highly pure nitrogen gas (flow rate of $50 \mathrm{~mL} / \mathrm{min}$ ) and that of $\mathrm{Q} 10$ was flushed with highly pure helium gas (flow rate of $25 \mathrm{~mL} / \mathrm{min}$ ). Temperature and enthalpy (cell constant) calibrations were based on the melting peak of the indium standard $\left(T_{\mathrm{m}}=156.6^{\circ} \mathrm{C}\right)$ supplied by TA Instruments, using the same experimental conditions (cell environment, type of pan/lid and heating rate) as for the samples.

X-ray powder diffraction (XRPD) experiments were performed with a PanAlytical X'PERT PRO MPD diffractometer ( $\lambda_{\text {cuKa }}=1.5418 \AA$ for combined $K_{\alpha 1}$ et $\left.K_{\alpha 2}\right)$ equipped with an X'celerator detector (Almlo, The Netherlands). Samples were placed into Lindemann glass capillaries (diameter $0.7 \mathrm{~mm}$ ) and installed on a rotating sample holder to avoid any artifacts due to preferential orientations of crystallites. Data were recorded from $5^{\circ}$ to $60^{\circ} 2 \theta$ by step of $0.0167^{\circ} 2 \theta$ using a counting time of 200 seconds per step. All experiments were carried out at room temperature. The instrumental resolution function, required for the microstructural analysis, was obtained by analyzing the XRPD pattern of standard compound $\left(\mathrm{Na}_{2} \mathrm{Ca}_{3} \mathrm{Al}_{2} \mathrm{~F}_{14}, \mathrm{NAC}\right)^{32}$. The XRPD patterns of as-received IBP, milled IBP and co-milled IBP:SBA-15[9nm] were analyzed using 
the Le Bail method ${ }^{33}$ included in FullProf ${ }^{34}$. The instrumental resolution function was obtained through a Le Bail whole-powder-pattern of a standard compound $\left(\mathrm{Na}_{2} \mathrm{Ca}_{3} \mathrm{Al}_{2} \mathrm{~F}_{14}, \mathrm{NAC}^{32}\right.$, in the $2 \theta$-angular range $7-60^{\circ}$. The Bragg peak profiles are fitted with the Thompson-Cox-Hastings pseudo-Voigt function ${ }^{35}$. "Profile matching" refinements ${ }^{33}$ were carried out from data obtained in CIF database (4512754). Raman investigations were performed using an InVia Renishaw Raman spectrometer (Renishaw plc, Wottonunder-Edge, Gloucestershire, UK). The 785 nm Renishaw diode laser beam was focused via a $\times 50$ long working distance Leica objective (Leica microsystemes, SAS, Nanterre, France), which allows to analyze a volume of about $30 \mu \mathrm{m}^{3}$ (the spot size being about $1 \mu \mathrm{m}$ in diameter and about $30 \mu \mathrm{m}$ in depth). The spectra were collected in backscattering geometry with a resolution of $2 \mathrm{~cm}^{-1}$ in the 20 $1200 \mathrm{~cm}^{-1}$ spectral range. The spectrometer is equipped of the holographic filter technology for investigations in the low-wavenumber region where lattice vibrations are detected. This technology requires the enhanced narrow band and low noise laser performance for the $785 \mathrm{~nm}$ laser source obtained using ASE filter systems.

Raman mapping was performed by scanning a $300 \times 300 \mu \mathrm{m}^{2}$ area point by point, with $4 \mathrm{~mm}$ step between points (for a total of 5776 spectra) and an acquisition time of 5 seconds per point. Mapping was carried out at room temperature. All spectra collected were baseline corrected before analysis with a polynomial function (degree = 1) and normalized in the region of collective motions $\left(20-200 \mathrm{~cm}^{-1}\right)$ using Wire 4.1 Matlab PLS Toolbox (Eigenvector Research Inc.) software. In a second step, the pure vibrational Raman intensity ( $\left.I_{\text {Raman }}^{\text {vib }}\right)$ is converted into Raman susceptibility $\left(\chi^{\prime \prime}(\omega)\right)$ according to the equation:

$$
\chi^{\prime \prime}(\omega)=\frac{I_{R a m a n}^{v i b}}{n(\omega)+1}
$$


where $n(\omega)=1 /\left(e^{\hbar \omega / k T}-1\right)$ is the Bose occupation number. For highly disordered systems, including amorphous states, $\chi^{\prime \prime}(\omega)$ can be expressed as:

$$
\chi^{\prime \prime}(\omega)=C(\omega) \cdot \frac{G(\omega)}{\omega}
$$

where $C(\omega)$ is the light-vibration coupling coefficient characterized by a nonuniversal wavenumber dependence. However, it has been shown ${ }^{36}$ that $\chi^{\prime \prime}(\omega)$ is a representation of the vibrational density of states (VDOS) close to that of $G(\omega)$ obtained by inelastic neutron scattering in the $5-200 \mathrm{~cm}^{-1}$, with a spectral resolution significantly higher than that of $G(\omega)$. Raman images can be calculated by fitting a linear combination of the reference spectra corresponding to crystalline and amorphous IBP in the undercooled liquid state, to all spectra collected at room temperature in the mapping procedure.

Drug release experiments were performed using a UV spectrometer (UV-1800 Spectrophotometer Shimadzu) in a wavelength range from 200 to $250 \mathrm{~nm}$, determined with respect to the absorbance peak at $220 \mathrm{~nm}$ of ibuprofen. Briefly, $14 \mathrm{mg}$ of IBP35\%:SBA-15[9nm] (4.9 mg of IBP confined into $9.1 \mathrm{mg}$ of MPS) were introduced in a flask containing $500 \mathrm{~mL}$ of $0.1 \mathrm{M}$ hydrochloric acid $(\mathrm{pH} 1.1)$ respecting Sink conditions $^{37,38}$. The ibuprofen amount was determined using the ibuprofen calibration curve performed in a $0.1 \mathrm{M}$ hydrocloric acid medium (Figure S2), in agreement with the literature ${ }^{39,40}$. The experiments were carried out at room temperature, under stirring conditions at $100 \mathrm{rpm}$, in order to always have all the particles in motion and avoid any deposit at the bottom of the container. For each measurement, $2 \mathrm{~mL}$ of solution were analyzed with the UV spectrometer and then poured back to the medium to avoid volume variations throughout the experiment. Experiments were replicated 6 times and the data presented are the mean with the standard deviation. The ibuprofen release 
percentage is determined by the ratio of the absorbance at a time $t$ over the absorbance at the time $t_{\infty}$, defined as the time for which it is assumed that all IBP molecules were released (measurement was carried out the next day). 


\section{Results and discussion}

\subsection{Thermal investigations of IBP population distribution in IBP:SBA-15[9nm]}

composites as function of the loading degree

\subsubsection{Thermogravimetric analysis (TGA)}

Thermogravimetric analysis is mostly used to evaluate the thermal stability and for determination of the guest content loaded in a mesoporous silica matrix. In IBP\%:SBA-15[9nm] composites the weight loss up to $550{ }^{\circ} \mathrm{C}$ is directly associated with the amount of adsorbed water and to the totality of confined and external IBP in cases where it remains also outside the mesopores (Supporting Information in $\operatorname{ref}^{26}$ ). It was shown for IBP:SBA-15[6nm] composites (35 $\leq$ IBP wt.\% $\leq 50)$ that there is a very good correlation between the theoretical (set during the sample preparation) and the experimental loading content achieved by the MAL method. In the present work, a TGA analysis of the prepared IBP:SBA-15[9nm] composites comprising a broad content range $(10 \leq \mathrm{IBP}$ wt. $\%<45)$ was carried out for characterizing the loading degree and, particularly, the weight loss profile related to the distribution of IBP molecules inside the SBA-15 mesoporous matrix.

Figure 1 illustrates the weight loss plots (Figure 1a) and their first temperature derivative (Figure 1b) for the composites corresponding to co-milling of 10, 20, 25 and 35 wt.\% of IBP with (100-IBP)wt.\% of SBA-15[9nm]. These plots highlight three components in their signals for the composites prepared with 20, 25 and 35 wt.\% of IBP:

- (i) from 20 to $75^{\circ} \mathrm{C}$, a component related to the evaporation of weakly physisorbed water molecules that probably were quickly adsorbed from the atmosphere during the handling required by the MAL method. 
- (ii) from 75 to $200{ }^{\circ} \mathrm{C}$, a component that can be attributed to evaporation of ibuprofen molecules located in the core of SBA-15[9nm] channels (IBP core population) ${ }^{26}$. Such straight assignment is possible due to the absence of a second peak (or shoulder) on the low temperature side of derivative signal, the only peak being observed at the same position for the bulk form and also observed for composites with IBP loadings higher than 35 wt.\%. This feature was previously reported ${ }^{26}$ for SBA-15[6nm] composites (IBP\% > 37 wt.\%) where a fraction of IBP crystals still remain outside the mesopores.

- (iii) from 200 to $550{ }^{\circ} \mathrm{C}$, a component associated with the evaporation of IBP molecules with higher thermal stability (relatively to bulk IBP) due to interactions with the pore walls (IBP surface population) ${ }^{26}$.

These results indicate that the distribution of IBP molecules inside the mesoporous SBA-15[9nm] can be described, in a simplified manner, as a bimodal distribution depending on the loading degree. The weight loss signals can be roughly decomposed as [\% core, \% surface] IBP components such as: [7\% core, $11 \%$ surface] for the composite loaded at $20 \mathrm{wt} . \%,[12.7 \%$ core, $12.7 \%$ surface] for the composite loaded at $25 \mathrm{wt} . \%$, and [22\% core, $13 \%$ surface] for the composite loaded at $35 \mathrm{wt} \%$. Repeated measurements using samples from the same MAL method batch or from different batches gave a reproducibility for the different loading components within \pm (1-2) wt.\% (the samples with the highest variations were those prepared with lower IBP contents). These results suggest that the contribution of IBP surface layer molecules can be considered the same for the three loading degrees (i.e., $\sim(12 \pm 1)$ wt. $\%$ for this system, IBP:SBA-15[9nm]) and, that during the encapsulation process using the MAL method, once the surface-layer is fully covered, additional IBP molecules will pile up in 


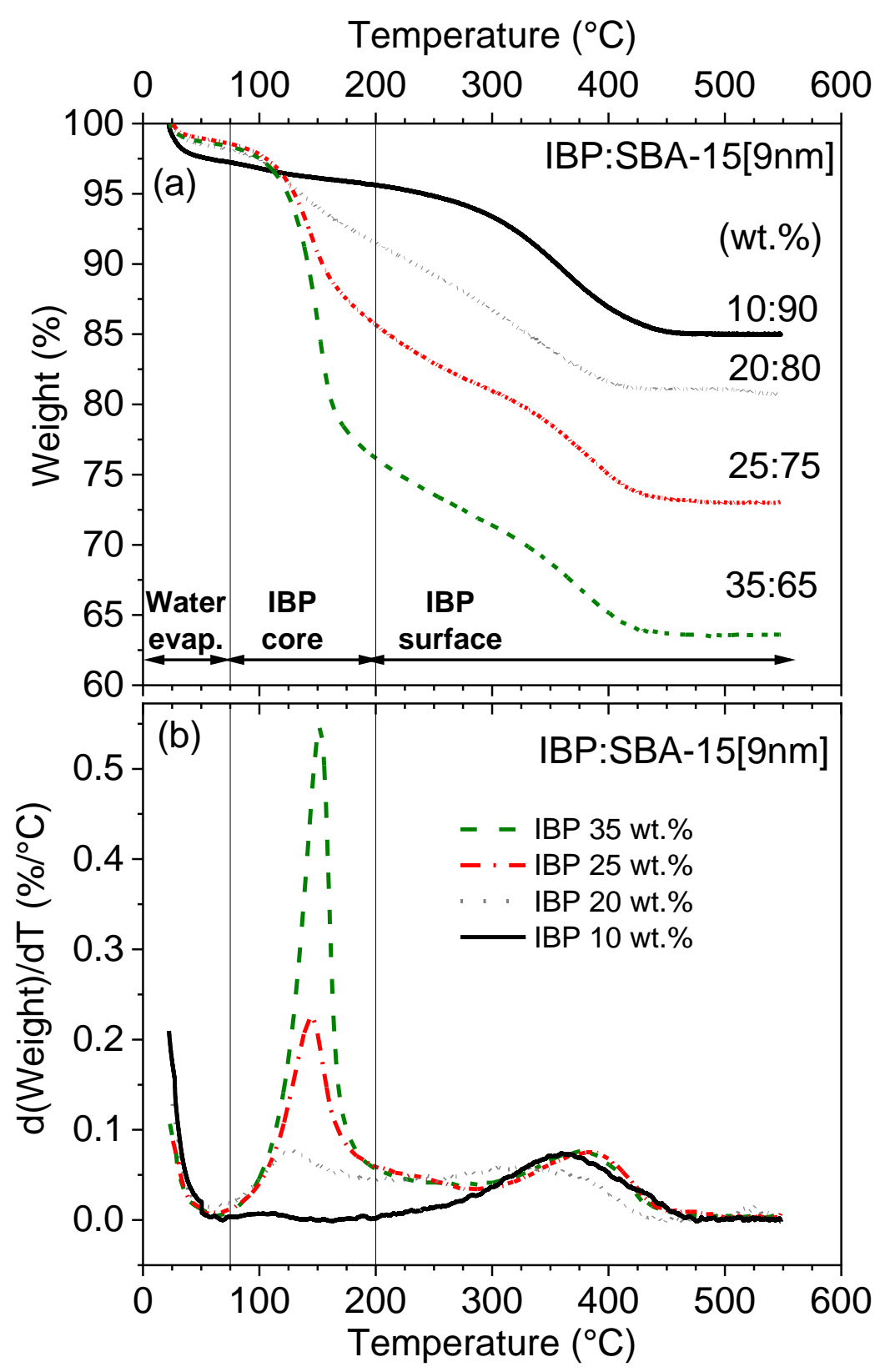

Figure 1. TGA curves obtained during heating at $5{ }^{\circ} \mathrm{C} / \mathrm{min}$ for four composites prepared by co-milling ibuprofen (IBP) with SBA-15[9nm] at indicated weight percentages. (a) weight loss plots and (b) first temperature derivative.

the core of the mesopores. For a composite loaded at 10 wt.\%, (black line in Figure 1a and $1 \mathrm{~b}$ ) the weight loss corresponding to the contribution of surface IBP molecules is around 10.6 wt.\%, which corroborates, within the precision achieved by the MAL method, the above conclusions for higher IBP loadings in SBA-15[9nm]. IBP10\%:SBA$\mathbf{1 5}[9 \mathrm{~nm}]$ composite presents yet a very small fraction of core IBP molecules (1.4 wt.\%) 
and, as expected, its content of physisorbed water molecules (2.8 wt.\%) is higher that of the higher IBP loading degree composites (1.6-1.8 wt.\%).

\subsubsection{Calorimetric analysis (DSC)}

3.1.2.1. Influence of the loading degree on the physical states and stability of IBP under confinement

Differential Scanning Calorimetry (DSC) is a very suitable technique to analyze the physical state, phase transformations and amorphous state stability against crystallization of the confined matter within MPS composites, by using the pertinent experimental protocol ${ }^{26}$. In a first procedure, DSC experiments were performed at heating rate of $1^{\circ} \mathrm{C} / \mathrm{min}$, on two IBP-SBA15[9nm] composites obtained using the MAL method with 35 and 40 wt.\% of IBP. The as-prepared composites were firstly cooled from room temperature down to $-80^{\circ} \mathrm{C}$ at $20^{\circ} \mathrm{C} / \mathrm{min}$ prior to the analysis along a first heating at $1^{\circ} \mathrm{C} / \mathrm{min}$ (run 1$)$ from $-80^{\circ} \mathrm{C}$ up to $100^{\circ} \mathrm{C}(\sim 25$ degrees above the melting temperature of bulk $\mathrm{IBP}^{41}$ ). After cooling down again (at $20{ }^{\circ} \mathrm{C} / \mathrm{min}$ ) to $-80{ }^{\circ} \mathrm{C}$, composites are re-analyzed along a second heating (run 2) identical to run 1. Ibuprofen milled for 30 minutes, under the same conditions used for the MAL of the composites, was also analyzed by DSC and it is used as reference for interpreting the results obtained for the IBP composites, given that SBA-15 matrix does not present any thermal event. The corresponding DSC traces, for bulk IBP and composites are plotted in Figure 2 (black solid lines for run 1 and red dash-dot lines for run 2).

Figure 2 a shows that, after cooling bulk IBP previously molten during run 1 , the glass transition is observed (run 2) at around $-50{ }^{\circ} \mathrm{C}$ through a $\mathrm{C}_{\mathrm{p}}$ jump distinctive of the solid-liquid transition, in agreement with previous works ${ }^{26,41}$. An exothermic peak 


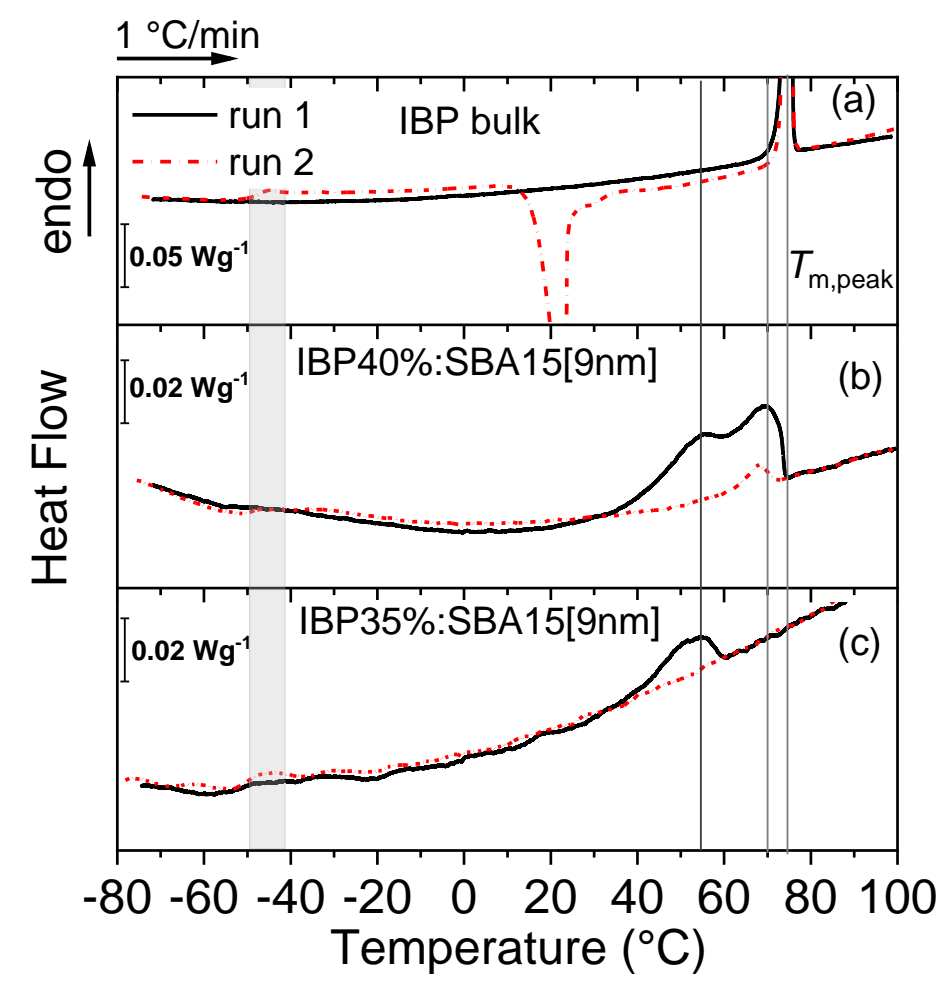

Figure 2. DSC curves recorded upon heating at $1{ }^{\circ} \mathrm{C} / \mathrm{min}$ (run 1, solid black line; run 2, dash-dot red line) for (a) bulk ibuprofen, (b) IBP40\%:SBA-15[9nm] and (c) IBP35\%:SBA-15[9nm] composite. Shaded gray area indicates the temperature interval of the glass-undercooled liquid transformation for bulk IBP and vertical lines indicate peak melting temperature ( $T_{m}$, peak).

characteristic of a cold-crystallization process is observed between 10 and $30^{\circ} \mathrm{C}$. It is followed by an endothermic peak at $75^{\circ} \mathrm{C}$ indicating that the undercooled liquid has recrystallized in Form I of $\mathrm{IBP}^{41,42}$. These results reflect the high instability of the amorphous state of bulk IBP at room temperature in agreement with previous studies $^{26,41}$.

The analysis of the IBP40\%:SBA-15[9nm] composite is presented in Figure $2 \mathrm{~b}$. The DSC trace of the first heating (run 1) highlights a broad and bimodal endotherm in the temperature range $\left[40^{\circ} \mathrm{C} ; 80^{\circ} \mathrm{C}\right.$ ]. The first component of this endothermic signal (with peak temperature, $T_{\text {peak }} \sim 55^{\circ} \mathrm{C}$ ) is observed at about 20 degrees below the melting peak of bulk IBP form I $\left(T_{\mathrm{m} \text {,peak }}=75^{\circ} \mathrm{C}\right)$ whereas the second component $\left(T_{\text {peak }}\right.$ $\sim 70{ }^{\circ} \mathrm{C}$ ) is very close to the melting of the bulk form. This suggests the existence of a broad size distribution of IBP crystallites in the as prepared IBP40\%:SBA-15[9nm] composite. A bulk-like crystalline fraction (with melting temperature $\sim T_{\text {m, bulk }}$ is 
coexisting with crystallites of smaller size characterized by lower melting temperatures $\left(<T_{\mathrm{m} \text {,bulk }}\right.$ ). At low temperature (gray shaded area in Figure 2$)$, a very subtle heat flow change around $T_{\mathrm{g}}$ (glass transition temperature) of bulk IBP mimicking the Cp jump of the glass transition is hardly observed. This attribution was confirmed by further experiments with higher heating rates, indicating the existence of amorphous IBP confined in the MPS by the MAL method, as previously observed ${ }^{26}$.

Upon the second heating (run 2), at temperatures above $T_{\mathrm{g}}$, the lower temperature component of the melting peak is no more observed. Only a small endotherm is detected at $\sim 70^{\circ} \mathrm{C}$, corresponding to the melting temperature of bulk-like IBP crystallites (form I) in the composite. This thermal event is much less intense than the corresponding peak in the as-prepared composite (run 1), that can have various origins. Firstly, it is clearly observed that both endotherms, especially the one detected at lower temperature, are very broad indicating the overlapping of melting endotherms of confined nanocrystals and unloaded crystal. It can be also considered that a part of the initial unloaded IBP crystallites does not recrystallize (after melting during run 1). This can be explained as a molten fraction of external IBP still penetrates the pores or/and it avoids crystallization due to interactions with the silanol groups of the outer surfaces of the pores. This event being very weak, its detection is also difficult due to a drift of the baseline that overlays the heat flow produced in the sample (the baseline drift is, as expected, less visible in the sample of bulk IBP (Figure 2a)).

Figure 2c shows the DSC thermograms of the IBP35\%:SBA-15[9nm] composite where no signature of bulk IBP crystallites outside the pores (melting peak at $70^{\circ} \mathrm{C}$ ) can be detected in both runs. This is the only distinctive feature compared to the DSC traces of the IBP40\%:SBA-15[9nm] composite shown in Figure 2b, showing that the maximum capacity of IBP loading in the SBA-15[9nm] matrix lies in between 
35 wt.\% and 40 wt.\%. During reheating (red dash-dot line in Figure 2c) coldcrystallization and melting are not observed, clearly indicating that crystallization, which occurs for the bulk form, is effectively suppressed by the nanoconfinement, as it was observed in IBP37\%:SBA-15[6nm] composite obtained by the MAL method ${ }^{26}$. The present results are the first to reveal the existence of nanosized crystallites for ibuprofen under confinement in a SBA-15[9nm] composite obtained by the Milling Assisted Loading method.

The IBP35\%:SBA-15[9nm] composite was analyzed using a second protocol, consisting in only increasing the scanning rate from 1 to $20^{\circ} \mathrm{C} / \mathrm{min}$, in order to get more information about the glass transition. An IBP25\%:SBA15[9nm] composite was also studied to get a better insight about the influence of the loading degree of IBP inside the pores, on the physical state and phase transformations. Bulk IBP was subjected to a similar thermal treatment for relevant comparison. All samples were heated twice as in the previous protocol (Figure 3a and 3c for bulk IBP and IBP35\%:SBA-15[9nm], respectively); the corresponding DSC traces are plotted in Figure 3 (black solid lines run 1 and red dash-dot lines - run 2).

Figure 3a shows that no recrystallization is detected upon heating the bulk amorphous form of IBP (run 2), and only the Cp jump with the overshoot related to the enthalpic relaxation, characteristic of the glass transition, is observed at $T_{\mathrm{g}}=-42$ ${ }^{\circ} \mathrm{C}$ (vertical line, $T_{\mathrm{g}}$ taken at the inflection point of the $\Delta \mathrm{C}_{\mathrm{p}}$ step). The crystallization of bulk IBP is avoided using a higher heating rate. As observed in Figure 3b, a clear detection of the glass transition signature in the IBP35\%:SBA-15[9nm] composite is now obtained (compared with Figure 2c). In the as-prepared 35\%-loaded composite (run 1, solid black line) the broad endothermic peak that was firstly reported for the 


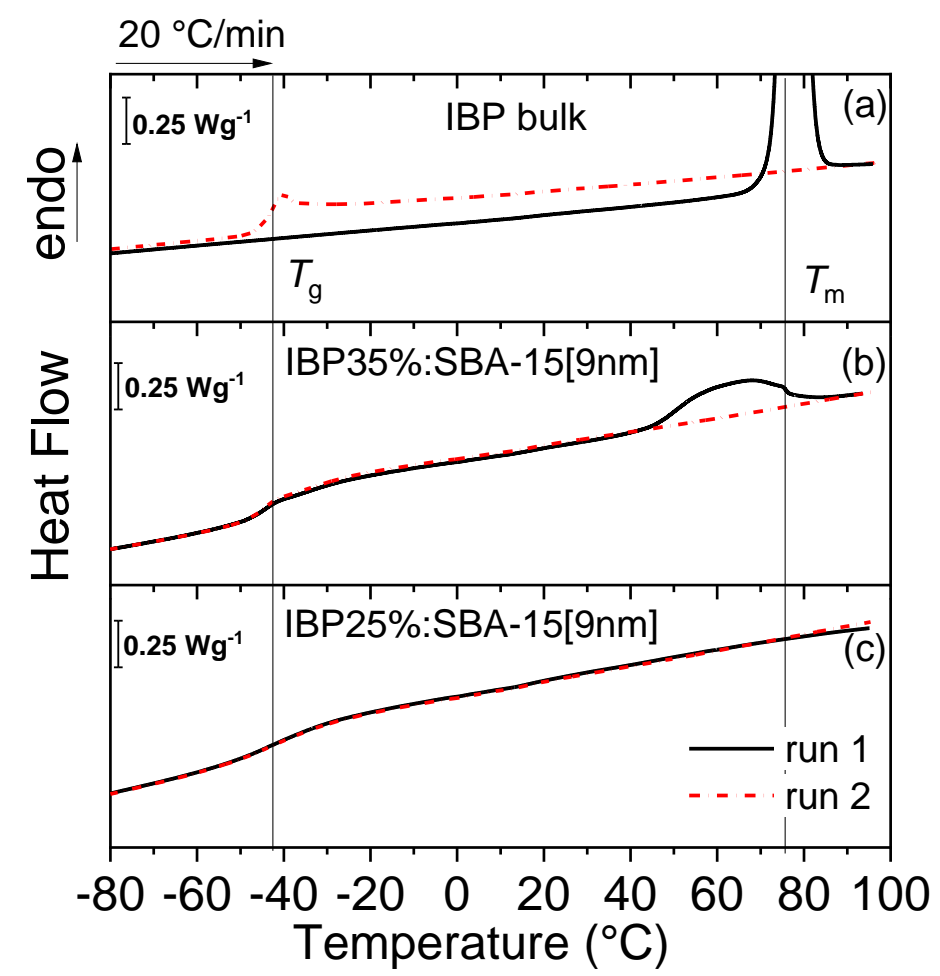

Figure 3. DSC thermograms recorded upon heating at $20{ }^{\circ} \mathrm{C} / \mathrm{min}$ (run 1, solid black line; run 2, dash-dot red line) for (a) bulk ibuprofen, (b) IBP35\%:SBA-15 and (c) IBP25\%:SBA-15 composite. Vertical lines indicate the glass transition temperature, $T_{g}$, and peak melting temperature, $T_{m}$ of bulk IBP.

IBP35\%:SBA15[9nm] sample analyzed with $\dot{T}=1^{\circ} \mathrm{C} / \mathrm{min}$ (Figure 2c) is also observed, in addition to the glass transition signature. This analysis confirms that comilling 35 wt.\% of IBP with 65 wt.\% of SBA-15[9nm] results in a composite containing amorphous and nanocrystallized regions. At $20^{\circ} \mathrm{C} / \mathrm{min}$, the melting endotherm ( $\mathrm{T}_{\text {peak }}$ $\left.=65^{\circ} \mathrm{C}\right)$ covers a relatively broader temperature range $\left(50^{\circ} \mathrm{C}<\mathrm{T}<75^{\circ} \mathrm{C}\right)$ than that $\left(\mathrm{T}_{\text {peak }} \sim 55^{\circ} \mathrm{C}\right)$ detected at $1^{\circ} \mathrm{C} / \mathrm{min}\left(40^{\circ} \mathrm{C}<\mathrm{T}<60^{\circ} \mathrm{C}\right)$. A close inspection of the melting peak of bulk IBP (Figure 2a and Figure 3a) also reveals an influence of the heating rate in the temperature range of the melting endotherm above $T_{\text {peak,bulk, reflecting }}$ thermal lag effects that are greater in the sample when the heating rate is higher. A crystallinity degree of around $8 \%$ is estimated for the IBP35\%:SBA15[9nm] composite, either from the evaluation based on the melting enthalpy values (ratio

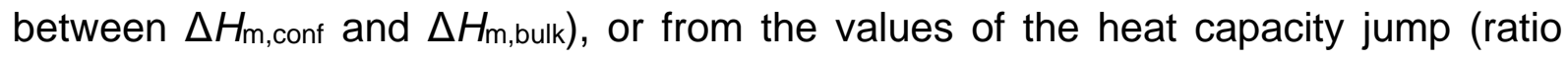
between $\Delta C_{\mathrm{p} \text {,run1 }}$ and $\Delta C_{\mathrm{p}, \text { run2) }}$ ) at $T_{\mathrm{g}}$, determined with the $20^{\circ} \mathrm{C} / \mathrm{min}$ heating rate 
procedure. Table 1 summarizes the thermodynamic parameters $\Delta C_{\mathrm{p}}, \Delta H_{\mathrm{m}}, T_{\mathrm{m}}$ of bulk IBP (after 30 minutes milling) and IBP35\%:SBA-15[9nm] composite.

Table 1. Heat capacity jump, $\Delta C_{\mathrm{p}}$ at the glass transition, melting enthalpy, $\Delta H_{\mathrm{m}}$, and melting temperature, $T_{m}$,peak, values obtained from DSC measurements carried out upon heating at $20{ }^{\circ} \mathrm{C} / \mathrm{min}$, for bulk IBP and IBP35\%:SBA-15[9nm] composite. Values between parentheses are the results from the analysis of the corresponding thermograms at $\dot{T}=$ $1{ }^{\circ} \mathrm{C} / \mathrm{min}$.

\begin{tabular}{|c|c|c|c|}
\hline Sample & $\begin{array}{c}\Delta C_{\mathrm{p}} \\
\left(\mathrm{J} / \mathrm{g} /{ }^{\circ} \mathrm{C}\right)\end{array}$ & $\begin{array}{c}\Delta H_{\mathrm{m}} \\
(\mathrm{J} / \mathrm{g})\end{array}$ & $\begin{array}{c}T_{\mathrm{m}} \text {, peak } \\
\left({ }^{\circ} \mathrm{C}\right)\end{array}$ \\
\hline Bulk IBP crystal (form I) - run 1 & - & 128 & $76(75)$ \\
\hline Bulk IBP vitrified - run 2 & $0.42(-)$ & $-(122)$ & $-(75)$ \\
\hline IBP35\%:SBA-15[9nm] - run 1 & $0.45(-)$ & $11(10)$ & $65(52-54)^{*}$ \\
\hline IBP35\%:SBA-15[9nm] - run 2 & $0.49(-)$ & - & - \\
\hline
\end{tabular}
temperature.

It can be noticed in Figure 3c that the glass transition is the only feature detected in the two thermograms (run 1 and run 2) of IBP25\%:SBA15[9nm]. No endothermic peak is observed in the first heating of the as-prepared composite, and necessarily both DSC heating traces are rigorously superimposed contrasting with the DSC traces collected by heating the IBP35\%:SBA15[9nm] composite. These results show that confined IBP by co-milling 25 wt.\% IBP with 75 wt.\% of SBA-15[9nm] is amorphous throughout the sample analyzed, while co-milling at a higher IBP content (35 wt.\%) results in a composite with both amorphous and nanocrystallized material. This clearly 
shows a direct influence of the density on the physical state of the confined matter in mesoporous SBA-15, only revealed using MAL method.

It is noteworthy that IBP is in a stable amorphous state without any trace of crystallization, when confined into IBP37\%:SBA-15[6nm] by $\mathrm{MAL}^{26}$, while in the present work, both crystalline and amorphous states are coexisting within IBP35\%:SBA-15[9nm] composite. The maximum loading capacity (37 wt.\% and 35 wt.\%) corresponds, in terms of filling degree to $~ 70(v / v) \%$ for the SBA-15[6nm] composite and $48(\mathrm{v} / \mathrm{v}) \%$ for the SBA15[9nm] composite (see SI for details of $(\mathrm{v} / \mathrm{v}) \%$ calculations). Considering the dimensions of the ibuprofen molecule ${ }^{4,43}, 1 \mathrm{~nm} \times 0.4 \mathrm{~nm}$ $\times 0.5 \mathrm{~nm}$, the average pore diameter of SBA-15[6nm] and SBA-15[9nm] correspond to roughly $6 \sigma$ and $9 \sigma$, respectively. Therefore, pore size effect most likely overcomes the density effect to explain the differences in the physical states of IBP confined in IBP37\%:SBA-15[6nm] and IBP35\%:SBA-15[9nm]. A more detailed analysis of the unusual coexistence of nanocrystalline and amorphous states of IBP in the IBP35\%:SBA-15[9nm] composite will be presented later in the text.

\subsubsection{Influence of the loading degree on the glass transition of IBP under confinement}

Using MAL method gives the opportunity to analyze in depth the glass transition from an enlarged view of the DSC traces around $T_{\mathrm{g}}$ presented in Figure $4 \mathrm{a}$. Both DSC traces corresponding to the two successive heating runs of the IBP35\%:SBA15[9nm] composite (top curves) reveal a double $C_{p}$ jump, in the same temperature range. This is the signature of two glass transitions of amorphous IBP under confinement, as previously observed for $\mathrm{IBP}^{26}$ and triton $\mathrm{X}-100^{44}$, both systems confined in SBA-15 matrices with $6 \mathrm{~nm}$ pore mean diameter. This double $C_{p}$ jump behavior has been also observed in different type of pores; ortho-terphenyl confined into disordered silica matrix $^{45}(C P G)$ or benzyl derivative of ibuprofen confined within aluminum oxide ${ }^{46}$ 
(AAO). The double $C_{p}$ jump was attributed to the glass transition of two populations of confined IBP molecules, located in the "core" and bound to the "surface" of channels, with distinct molecular mobility inside the pore $24,25,44$. The "core" population is characterized by a molecular mobility enhanced with respect to that in the bulk amorphous form (lower $T_{\mathrm{g}}$ ), while interactions between the "surface" population and the inner surface of the pores induce the slowing down of the mobility (higher $T_{\mathrm{g}}$ ). 


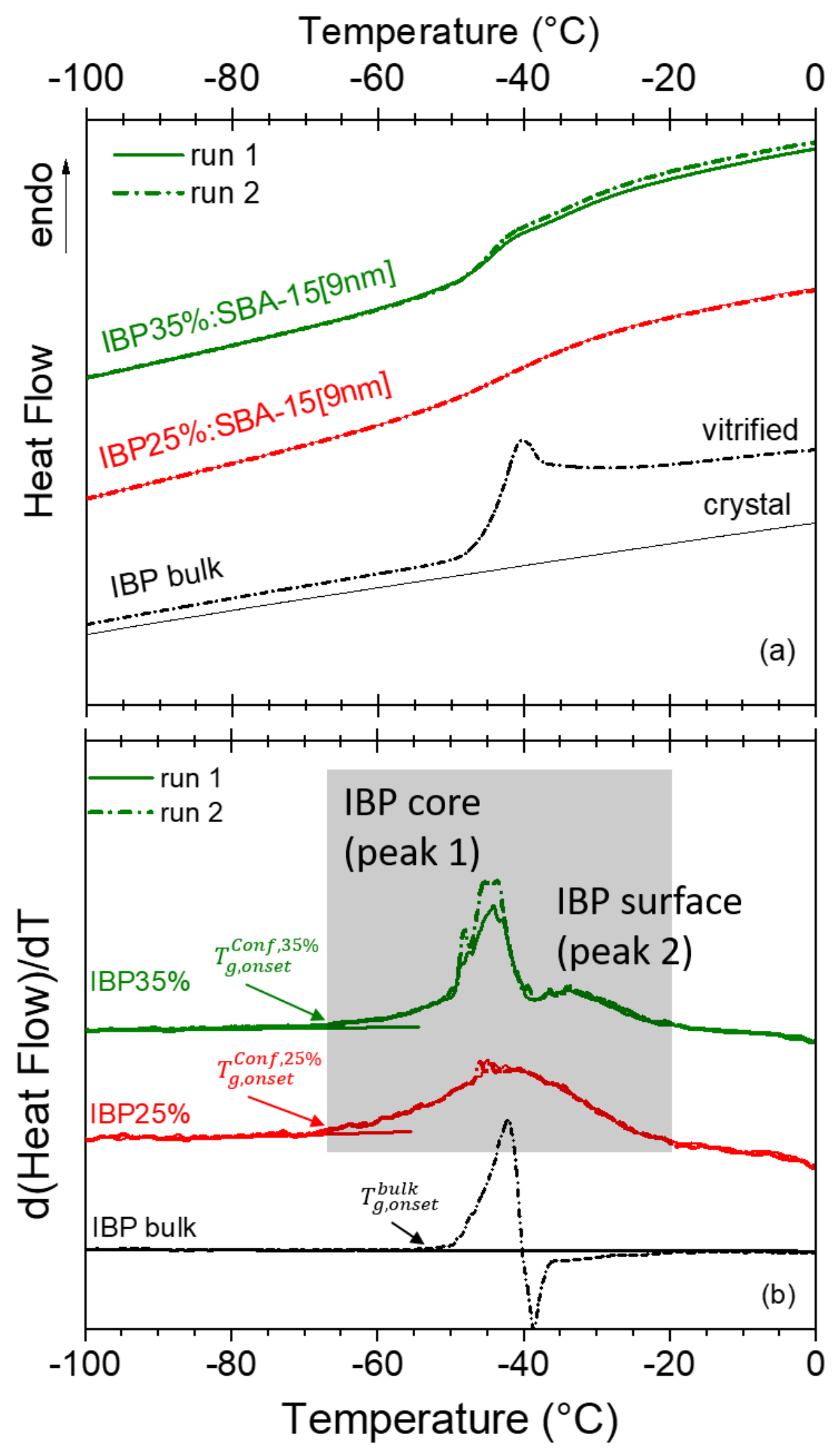

Figure 4. Enlarged view of the glass transition region shown in Figure 3. (a) Heat flow and (b) first temperature derivative of heat flow of curves shown in (a). Curves vertically shifted for clarity. 
Considering this simplified two-population model, also called two-state model ${ }^{47}$, the thickness of the surface layer, $\delta$, can be estimated ${ }^{48}$ :

$$
\delta=\frac{d}{2} \times\left[1-\left(1-\frac{\Delta C_{p, \text { surface }}}{\Delta C_{p, \text { total }}}\right)^{1 / 2}\right]
$$

where $d$ is the pore diameter, and the total heat capacity $\Delta C_{p, \text { total }}=\Delta C_{p, \text { core }}+$ $\Delta C_{p, \text { surface }}$. In this estimate, it was assumed, (i) fulfilled cylindrical geometries and (ii) $\Delta C_{p, \text { core }}, \Delta C_{p, \text { surface }}$ are considered proportional to the number of molecules of each population ${ }^{48}$. The calculations must be performed using the second run to get the total $\Delta C_{p, \text { core }}$, since a part of the core population is crystallized in the first heating run (Figure 3b). The thickness of the disordered surface layer estimate using equation (1) is $\delta=$ $(1.15 \pm 0.15) \mathrm{nm}$, averaged from calorimetric data of replicated measurements (Figure S3). This estimation agrees well with values reported for ibuprofen confined in porous silicon (1.2-2 nm $)^{20}$ and in controlled pore glass $(1-3 \mathrm{~nm})^{18}$. It is interesting to note here that in the studies mentioned, the thickness of the surface layer was calculated from calorimetric data based on the melting enthalpy of IBP nanocrystals. Indeed, in the composites of those studies, the only amorphous contribution came from molecules located at the pore walls, i.e., the surface layer. The estimate thickness of the surface layer is close to the length of the ibuprofen molecule ${ }^{43,49}$. The present DSC results also clearly reveal the existence of a non-crystallizable ibuprofen monolayer between the silanol groups of the silica walls and ibuprofen nanocrystals in the SBA-15[9nm] mesopores.

The first temperature derivative of the heat flow signal $(\mathrm{dHF} / \mathrm{dT})$, plotted in Figure $4 b$, is used for accurately determining the transition limits and for splitting the 
two overlapping spread $\mathrm{C}_{\mathrm{p}}$ jumps into two peaks, allowing thereby a better insight on the glass transition ${ }^{50}$.

For the IBP35\%:SBA15[9nm] composite, the first derivative of the double $C_{p}$ jump results in two peaks clearly detected (top curves) covering a temperature range of almost 50 degrees (gray shaded area). The first peak (1) observed at $T_{\text {g,peak } 1=-45}$ ${ }^{\circ} \mathrm{C}$ has a pronounced tail lying between $-70^{\circ} \mathrm{C}$ and $-50^{\circ} \mathrm{C}$, contrasting with bulk IBP (black bottom dash-dot curve) for which a single and narrow peak at $T_{\text {g,peak bulk }}=-42$ ${ }^{\circ} \mathrm{C}$ is observed. Peak 1 maximum is detected slightly below that of IBP bulk. This decrease of $T_{\mathrm{g}}$ was previously interpreted as reflecting the size reduction of domains where molecules rearrange in a cooperative manner $(C R R)^{51}$, inducing increasing molecular mobility (lower $T_{\mathrm{g}}$ ). The pronounced tail on the low-temperature side of peak 1 is likely induced by a size distribution of CRR. The second peak at $T_{\text {g,peak } 2}=-35^{\circ} \mathrm{C}$ (see also Figure S3) is associated with the surface population, in interactions with silanol groups inducing thereby a slower mobility.

Upon the second heating (dash-dot line) after liquid quench of confined ibuprofen in the IBP35\%:SBA15[9nm] composite, the only change in the first derivative trace results in the intensity increase of peak 1. Anywhere else the two traces of the first and second heating runs are rigorously superimposed. This indicates that the melting of the confined crystalline matter has been vitrified into a glassy state similar to that of the core population in the as-prepared composite. This feature is observed for several replicated experiments on different batches of IBP35\%:SBA15[9nm] composites (Figure S3). This result agrees with the previous description of an amorphous layer interacting with the surface, surrounding a core population composed of coexisting nanocrystalline-amorphous states or merely amorphous state with higher molecular mobility. 
In the IBP25\%:SBA15[9nm] Figure 4a clearly reveals a single $\mathrm{C}_{\mathrm{p}}$ jump (middle curves). Figure $4 \mathrm{~b}$ shows that only one broad and asymmetric peak is observed covering the trace of the 2 peaks in the IBP35\%:SBA15[9nm] composite. This indicates that there is no most representative population between core and surface population, probably because of the relative dispersion of the molecules in the core. It must be noted that a binary model corresponding to a low density of molecules in the surface layer and a high density of molecules in the core is the main limitation of a more likely continuous density-gradient real situation.

\subsection{Analysis of the nanocrystalline-amorphous coexisting states of IBP in the}

\section{IBP35\%:SBA-15[9nm] composite.}

No evidence of confined ibuprofen in a nanocrystallized stable state has yet been mentioned in the literature for SBA-15 mesoporous matrixes of pore diameter equivalent to the one used in the present study (i.e. average pore diameter $D_{p, d e s}=9.4$ $\mathrm{nm}$ determined by $\mathrm{BJH}$ from desorption branch and $\mathrm{D}_{\mathrm{p} \text {,ads }}=11.4 \mathrm{~nm}$ from adsorption branch). However, comparisons with data reported in the literature require consideration of various parameters (loading methods, loading degrees or methods to determine the pore diameter) which have a direct influence on the physical state of confined matter. Mellaerts et al. ${ }^{23}$ have not detected any trace of crystallization for ibuprofen confined by different liquid imbibitions (from the melt or dissolved in a solvent) into SBA-15 with $D_{p, a d s}=8.4 \mathrm{~nm}$ pore diameter at a loading degree of $30 \mathrm{wt} . \%$. Similar results were reported by Izquierdo-Barba et $a .^{24}$ and Brás et $a l .^{25}$, for comparable SBA-15 pore diameters and loading degrees $\left(D_{p, a d s}=8.8 / 8.6 n m ; \sim 20\right.$ wt.\% loading). Shen et al. ${ }^{52}$ reported no confined crystalline state of ibuprofen loaded by co spray-drying inside SBA-15 with a pore diameter of $D_{p, a d s}=6 \mathrm{~nm} \quad$ (46 wt.\% loading degree), while they observed nanoconfined ibuprofen crystals with amorphous 
IBP inside SBA-15 with $D_{p, \text { ads }}=20 \mathrm{~nm}$ (48 wt.\% loading degree). More recently ${ }^{26}$, no trace of crystalline IBP was detected in SBA-15 $\left(D_{p, \text { des }}=6 \mathrm{~nm}\right)$ loaded by the MAL method at a loading degree of $37 \mathrm{wt} . \%$. Similarily, Riikonen et al. ${ }^{20}$ have reported traces of crystallization for ibuprofen confined by solution imbibition into mesoporous silicon (loading degree of 20 wt.\%) with an average pore diameter $D_{p, \text { des }}=11.3 \mathrm{~nm}$ slightly higher than the $D_{p, d e s}=9 \mathrm{~nm}$ of our SBA-15 (the pore diameter distribution of the mesoporous silicon is also wider than SBA-15). In order to perform a robust statement on the influence of MAL method on the physical state of the confined matter, loading IBP by impregnation from solution in SBA-15[9nm] was carried out. No signature of nanocrystals was found in the IBP35\%:SBA15[9nm] composite obtained from a solution imbibition (Figure S4a), only the double glass transition $C_{p}$ jump is observed (Figure S4b) clearly indicating that MAL method promotes crystalline state in SBA$15[9 \mathrm{~nm}]$ matrix. Thus, this points out a unique situation for which the solid-state loading method is responsible.

\subsubsection{Insights on nanocrystals size distribution from calorimetric results}

The DSC results discussed in the previous section clearly revealed the signature of nanocrystalline-amorphous stable states (degree of crystallinity $8 \%$ ) of IBP confined in SBA-15 with an average pore diameter, $\mathrm{D}_{\mathrm{p} \text {,ads }}$ (BJH adsorption branch), of $11.4 \mathrm{~nm}$. The melting peak is located at 23 degrees below the bulk (Figure 2 and Table 1) which represents a higher melting point depression than the one calculated for IBP confined in SBA-15 with $D_{p, a d s}=20.1 \mathrm{~nm}$ (melting depression of $\sim 14$ degrees) reported in the work by Shen et al. ${ }^{52}$ This size effect on the melting transition is only related to the pore diameter for confined crystallites ${ }^{9,53}$. Shimizu et al. ${ }^{54}$ determined the dependence of the melting temperature to the pore diameter for ibuprofen confined in Controlled Pore Glass (CPG) with average pore diameters within the range [38 nm; 
$300 \mathrm{~nm}$ ]. For that, a flow column-based loading process was used to first load IBP into the $\mathrm{CPG}$ and then crystallize it in the pores through evaporation ${ }^{55}$ (in this paper, a maximum loading degree of around 33 wt.\% ibuprofen in CPG was achieved). A decrease of the melting temperature with reduction of pore diameter was also reported by Riikonen et al. ${ }^{20}$ for IBP inside mesoporous silicon (PSi) ranging from $11 \mathrm{~nm}$ to 75 $\mathrm{nm}$ (BJH desorption branch). The melting temperature data of confined IBP as function of the diameter of pores is summarized in Table $\mathrm{S} 1$ (supplementary material).

The relationship between the magnitude of the melting temperature depression, $\Delta T_{\mathrm{m}}$ and the inverse of pore diameter, $d$, is usually described by the Gibbs-Thomson equation, assuming cylindrical shape of the crystals ( $\operatorname{ref}^{9}$ and references therein):

$$
\Delta T_{m}=T_{m}-T_{m}(d)=\frac{4 \sigma_{s l} T_{m}}{d \Delta H_{m} \rho_{s}}
$$

where $\sigma_{s l}$ is the surface free energy of the solid-liquid interface, $T_{\mathrm{m}}$ is the melting temperature in the bulk form, $T_{\mathrm{m}}(d)$ is the melting temperature of crystals within a constant pore diameter of size $d, \Delta H_{m}$ is the melting enthalpy and $\rho_{s}$ is the density of the solid. In this thermodynamic relationship, the diameter of the confined crystal is considered equal to the pore diameter, which is actually an upper limit on the crystal diameter ${ }^{39}$. In addition, it is often assumed that $\sigma_{s l}$ is isotropic and the crystal size is sufficiently large so that the material retains its bulk properties ${ }^{9,53}\left(\Delta H_{m, b u l k}\right.$ and $\left.\rho_{s}\right)$. 


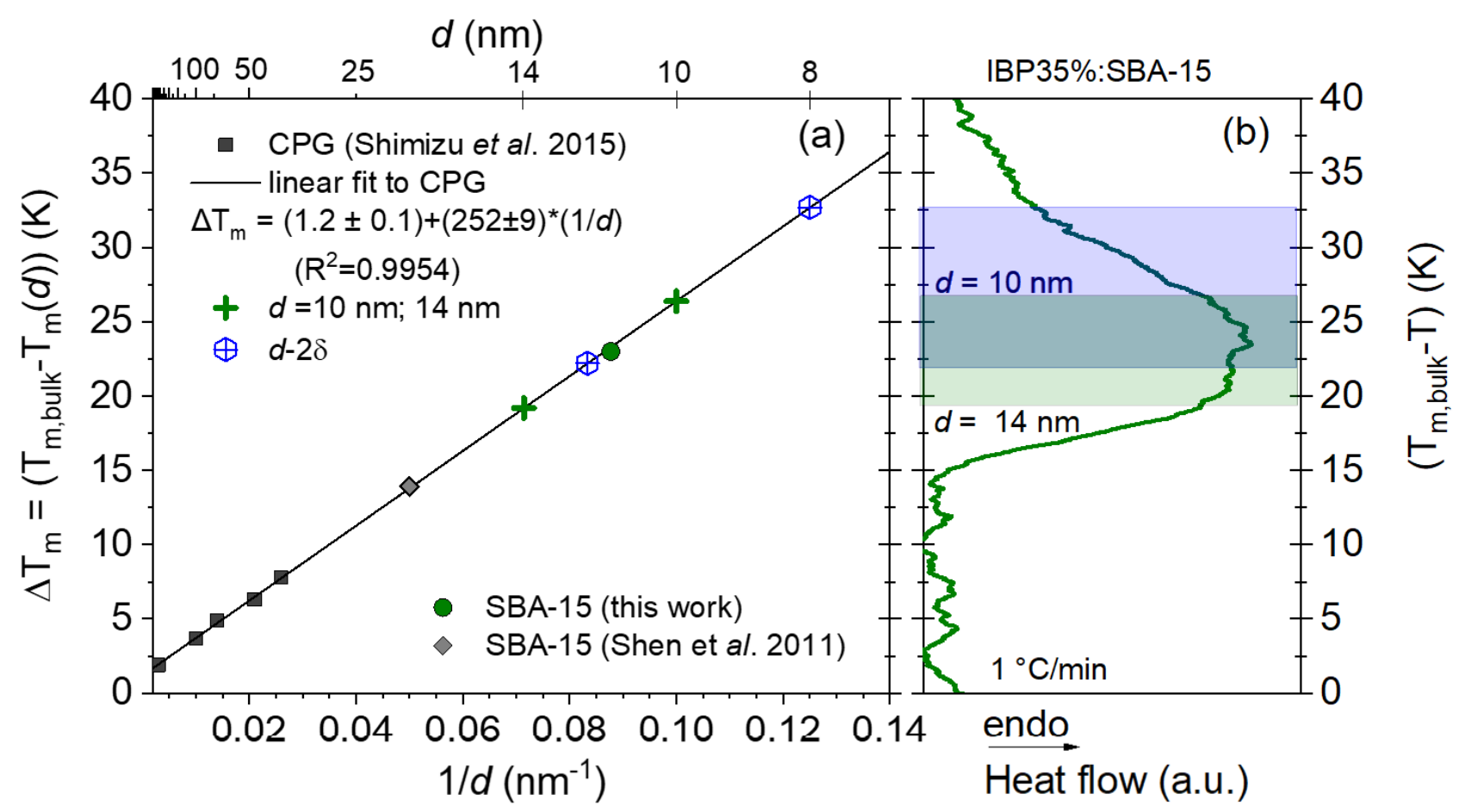

Figure 5. (a) Plot of melting temperature depression of confined ibuprofen, $\Delta T_{m}$, as a function of the inverse of the pore size, $1 / d$, where $d$ is the average pore diameter calculated in the BJH adsorption branch. Data of IBP:SBA-15 composites: this work (olive circle) and literature (gray lozenge); IBP:CPG composites of several sizes from literature (black squares). (b) DSC curve recorded at $1^{\circ} \mathrm{C} / \mathrm{min}$ for the IBP35\%:SBA-15 composite (Figure 2c); the SBA-15 matrix used in the present work has average pore diameter in BJH adsorption branch of $11.4 \mathrm{~nm}$ and pore size distribution [10 $\mathrm{nm} ; 14 \mathrm{~nm}$ ]. The liquid layer can be considered, reducing the crystallite sizes from $d$ to $d$ $2 \delta$ (blue crossed hexagons). See text for details.

Figure 5 a shows the melting temperature depression, $\Delta T_{m}=\left(T_{m, b u l k}-T_{m}(d)\right)$, plotted against the inverse of the pore diameter $(d)$. The straight line (black line) is the linear fit to the data obtained for IBP confined in $\mathrm{CPG}^{38}$ (full squares). The two points that represent IBP confined in SBA-15 (present work (full circle) and literature ${ }^{52}$ (full lozenge) are well aligned with the CPG data. $\Delta T_{m}(1 / d)$ data of IBP inside PSi follow almost the same trend (Figure S5) but some deviations are observed because the pore diameter was calculated from the BJH desorption branch ${ }^{20}$. Figure 5 a shows that confined nanocrystals of IBP, in various matrixes (ordered (SBA-15) and disordered (CPG), pore diameter range, related to much different melting enthalpy values ${ }^{20}$ ) exhibit the linear dependence between $\Delta T_{\mathrm{m}}$ and $(1 / \mathrm{d})$ predicted by the Gibbs-Thomson relationship. From the knowledge of the pore diameter distribution of the SBA-15 used here (i.e. [10 nm; $14 \mathrm{~nm}$ ] Figure S1), reporting the extreme values (cross points) in Figure $5 \mathrm{a}$ makes possible to extrapolate the $\Delta T_{\mathrm{m}}$ values corresponding to each extremity. The temperature range (green shaded area in Figure 5b) determined by these values is plotted with the DSC trace corresponding to the first heating run of the IBP35\%:SBA-15 composite. It is observed that this temperature range is well fitting 
with that corresponding to the intensity maximum of the broad melting endotherm confirming thereby that the size effect is imposed by the channel diameter, not by the crystallite size necessarily developing along the axis of cylindrical channels (micrometric size) ${ }^{53}$. To get a better understanding of this very broad melting endotherm, the layer remaining liquid at the inner surface of the pores can be considered, thereby reducing the crystallite size from $d$ to $d-2 \delta$. The new temperature range (blue shaded area in Figure $5 b$ ) corresponds to the low temperature tail of the melting event. This low temperature tail is most likely the manifestation of the reduction of crystallites size due to the liquid layer.

\subsubsection{X-ray powder diffraction analysis}

The physical state of IBP within pores was analyzed from X-ray diffraction and low-wavenumber Raman investigations performed in the IBP35\%:SBA-15[9nm] composite in order to only analyze of the crystallites within the pores.

Figure 6 presents X-ray powder diffraction (XRPD) patterns of bulk IBP in form I (as received), IBP milled for 30 minutes and the IBP:SBA-15[9nm] composite with a 35 wt.\% loading degree, plotted in the $2 \theta$ region lying between 12 and $27^{\circ}$ where the most intense Bragg peaks of form I are detected. The XRPD pattern of the composite shows Bragg peaks emerging from the intense and diffuse halo related to the SBA15[9nm] matrix and amorphous IBP inside the pores. The pattern of nanocrystals confined within the pores was obtained after removing the amorphous contribution, and plotted for relevant comparison with XRPD patterns of as-received and milled IBP. These peaks coincide with the peaks of the crystalline form I of ibuprofen. The ordered hexagonal organization of channels gives Bragg peaks at very small $2 \theta$ values ${ }^{56}$, not detectable with our equipment. "Profile matching" refinements ${ }^{33}$, carried out from data obtained in CIF database (4512754), have led to determine the $2 \theta$-dependence of full width at half maximum (FWHM) plotted in Figure S6 only for as-received and milled IBP. No convergence can be reached for the composite, because of the lack of robust 


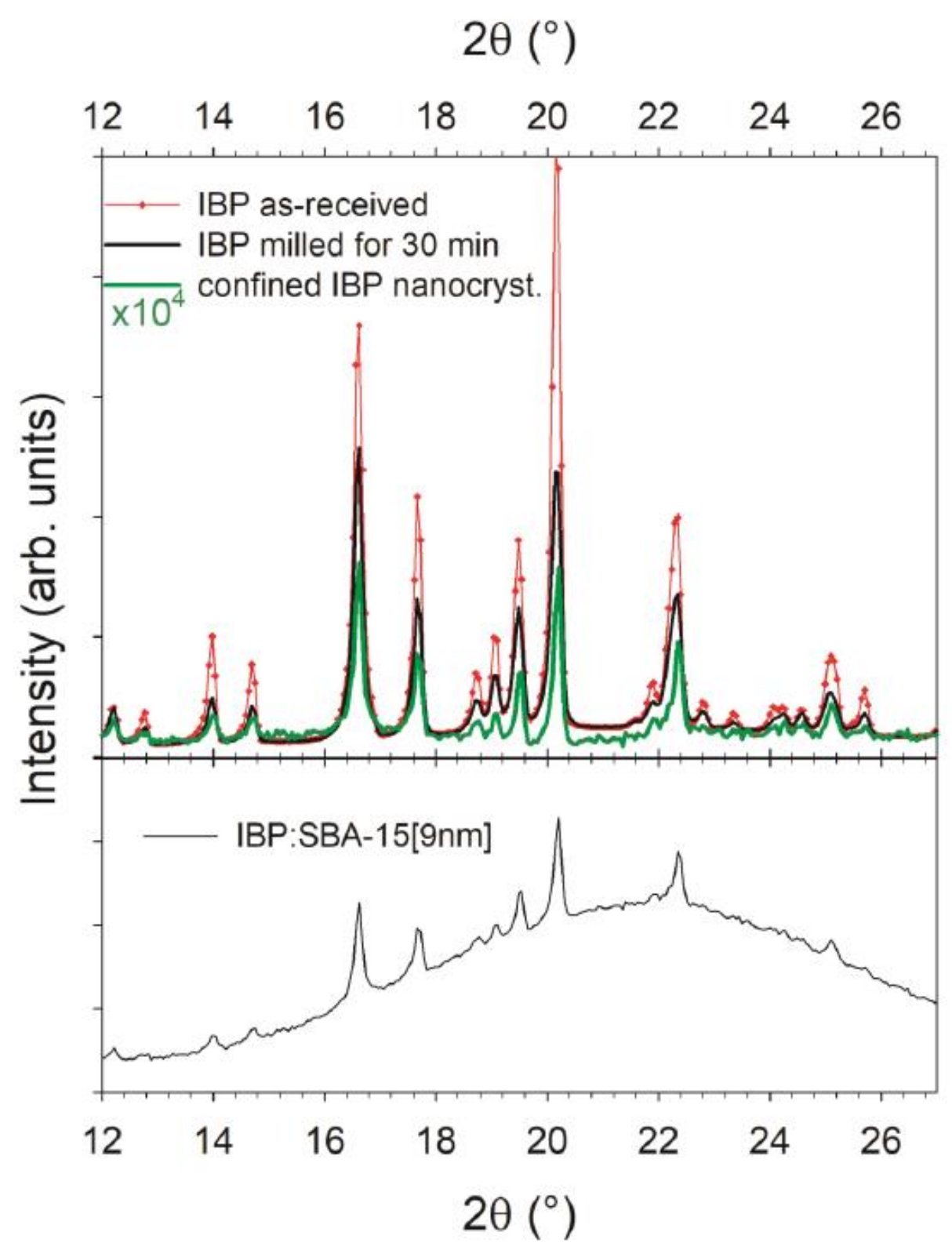

Figure 6. XRPD patterns of IBP in the top graph (as-received, milled and confined nanocrystals), and pattern of the IBP35\%:SBA-15[9nm] composite in the bottom graph.

data. Indeed, Figure 6 clearly shows that only a few Bragg peaks can be detected corresponding to the most intense peaks of the as-received IBP pattern. Figure S6 shows that FWHM of as-received IBP and milled IBP vary as the inverse of $\cos \theta$, typical of size effect. The size of spherical crystallites (isotropic model) was estimated as $691 \AA$ in as-received IBP and $488 \AA$ after milling. This typical mean size is considerably larger than the average pore diameter of $94 \AA$. Hence, during the loading process by milling-assisted method, crystallites must be strongly pressurized to force the inclusion of ibuprofen molecules inside channels. 


\subsection{3. $\mu$-Raman mapping analysis}

A more detailed description of the physical state of confined IBP in the IBP35\%:SBA-15[9nm] composite can be obtained by $\mu$-Raman mapping. A $300 \mu \mathrm{m} \times$ $300 \mu \mathrm{m}$ area was scanned, by collecting spectra in the $20-200 \mathrm{~cm}^{-1}$ low-wavenumber spectral region. Fitting each spectrum by a linear combination of crystalline Form I and amorphous spectra normalized in the spectral of collective motions directly provide a degree of crystallization estimated from the color scale. The Raman image obtained by fitting the 5776 spectra collected in the mapping procedure is presented in Figure 7. Spectra characterizing specific regions are plotted in Figure 8. The Raman image highlights a strong dark (blue) dominance reflecting a low degree of crystallization of confined IBP, and also reveals an inhomogeneous distribution of the physical state of confined IBP. It is noticeable that no lattice modes can be detected in blue regions indicating the amorphous character of IBP. By contrast, light (yellow) regions are characterized by spectra close to that of form I of IBP with the clear observation of

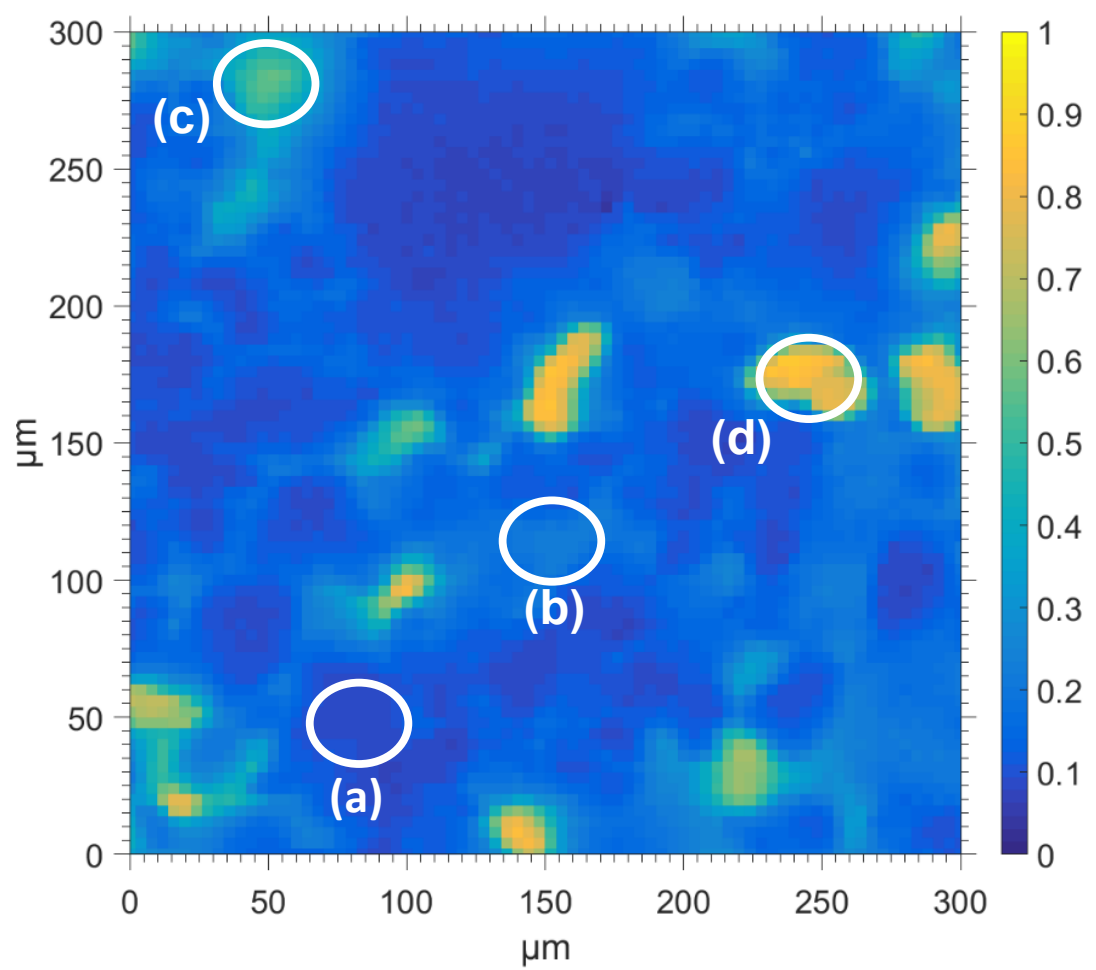

Figure 7: Raman image of the crystallinity degree (blue for the amorphous and yellow for the crystal) of an asprepared IBP35\%:SBA-15[9nm] composite. 
phonon peaks in agreement with the observation of Bragg peaks by XRD. Intermediate states corresponding to nano/micro crystals are also observed via a variety of lowwavenumber band-shapes in Figures 8. This physical state distribution can be interpreted as (i) inhomogeneous crystallites size along the channel length and (ii) different physical states between neighboring pores. The analyzed volume of each area represented by a pixel on the Raman image probes several thousand mesopores (spot diameter: $\sim 1 \mu \mathrm{m}$, pore diameter: $\sim 10 \mathrm{~nm}$ ). This classical analysis allows estimating the crystalline degree as about $15 \%$. This result, slightly different from that obtained by DSC investigations (8\%), can be explained by a sub-sampling effect inherent to the volume analyzed by Raman mapping significantly smaller than that analyzed by DSC (about $30 \mu \mathrm{m}^{3}$ and $40 \mathrm{~mm}^{3}$, respectively). 


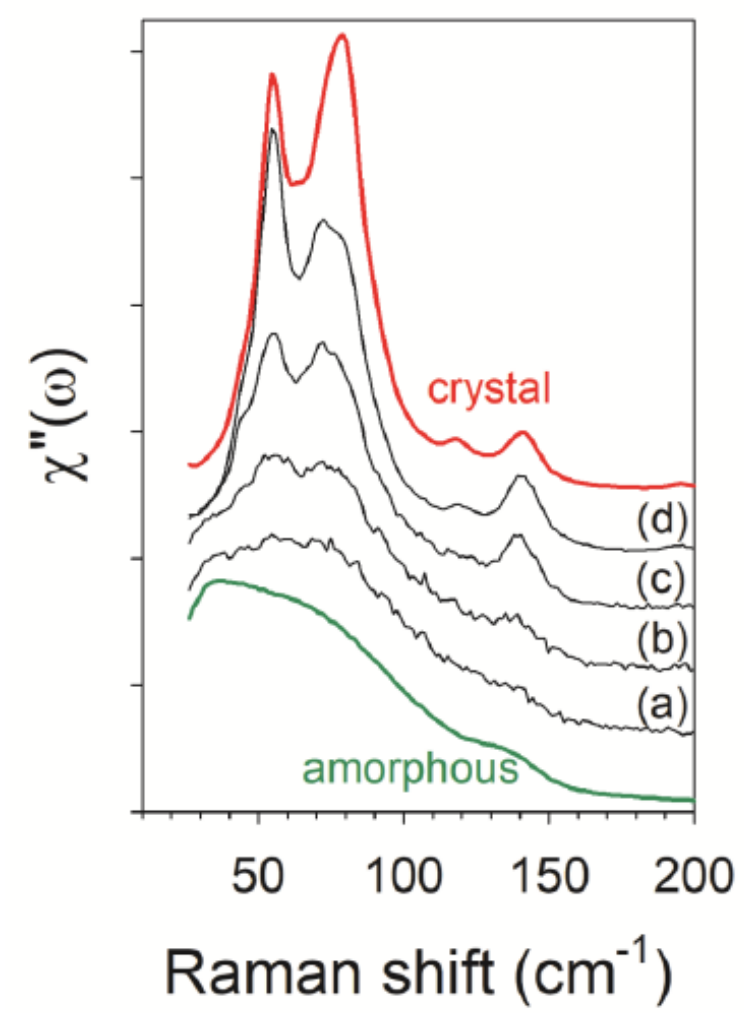

Figure 8 : Low-wavenumber Raman spectra collected in the spatial regions (a), (b), (c) and (d) located by the same respective letters in the Raman image in Figure 7 of IBP35\%:SBA-15[9nm] composite. Spectra distinctive of these four regions are compared with those of the undercooled liquid (bottom olive curve) and crystalline form I (top red curve) of bulk IBP.

\subsubsection{Physical state influence on release profiles of IBP within IBP35\%:SBA-15[9nm]}

composite

The release profile of ibuprofen from the IBP35\%:SBA-15[9nm] composite was studied to determine the influence of the physical state of confined ibuprofen. For that, two samples of the same composite batch were taken for release experiments carried out in acidic medium and sink conditions (details in experimental section). One sample corresponds to the as-prepared composite, where IBP is present in amorphous and nanocrystalline states, i.e, sample named here IBP35\%:SBA-15[9nm] "with nanocrystals" (crystallinity degree $~ 8 \%$ ). The second one consists of IBP35\%:SBA$15[9 \mathrm{~nm}]$ composite that was previously heated at $80^{\circ} \mathrm{C}$ in order to melt the confined nanocrystals and cooled to room temperature before the release profile measurements. The latter sample is identified as IBP35\%:SBA-15[9nm] "with melted 
nanocrystals". The results of release measurements of IBP from these two samples are shown in Figure 9 (olive circles and triangles), and compared to the dissolution of the bulk crystal form I (black squares).

Figure 9 shows a rapid ibuprofen release from the two IBP35\%:SBA-15[9nm] composites compared to the dissolution of the bulk crystal form I. This difference is related to the physical state of the ibuprofen within the composites which is mostly amorphous (or fully in case of "melted nanocrystallites" sample) and the solubilization of ibuprofen does not require to provide energy to destroy the crystal lattice.

Ibuprofen release profiles from the two studied samples of IBP35\%:SBA$15[9 \mathrm{~nm}]$ composite (as-prepared and after melting of nanocrystals) both revealed the same qualitative scenarios: (i) a very fast first release stage, the so-called "burst effect"57 followed by (ii) a very slow release of IBP in a second stage. These two stages were already reported in the literature for IBP confined in different mesoporous

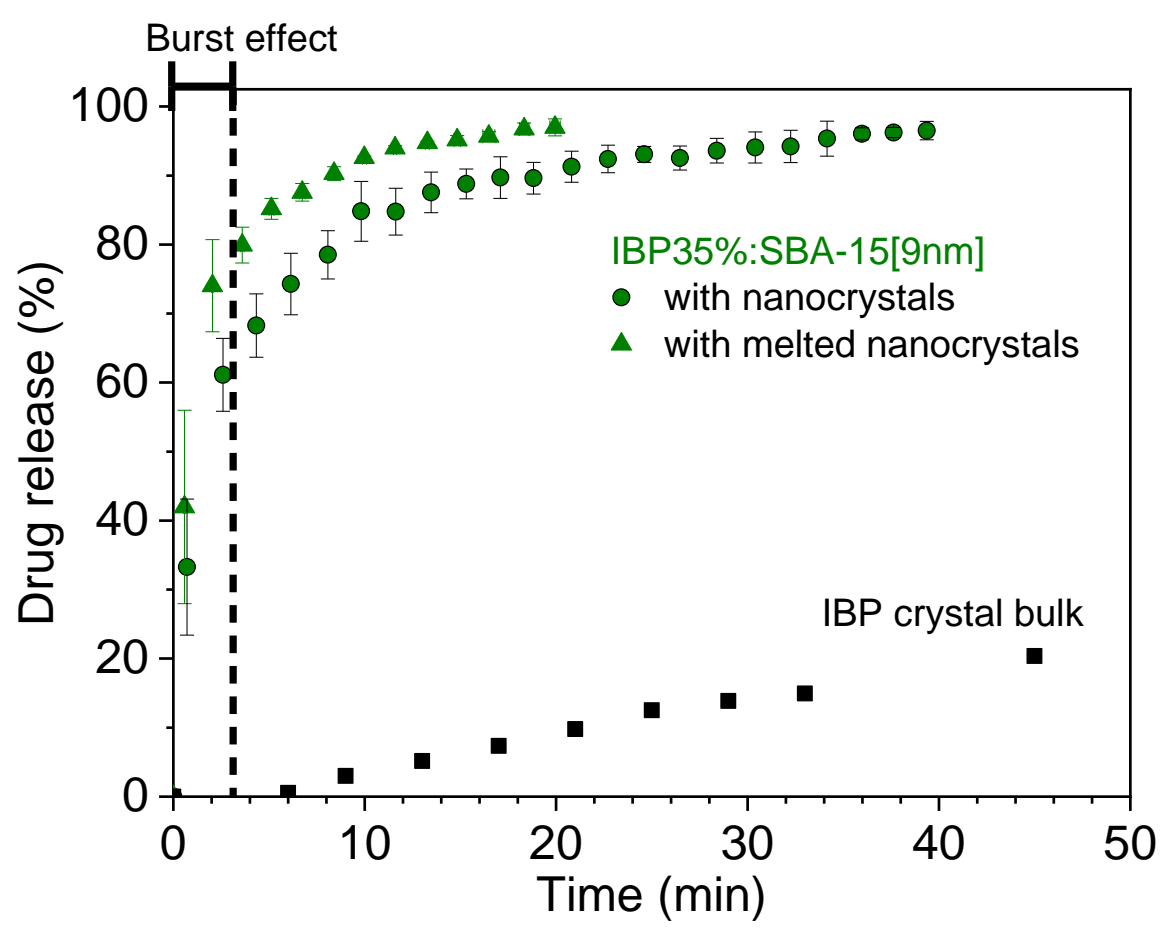

Figure 9. Release/dissolution profiles of ibuprofen from two samples of IBP35\%:SBA-15[9nm] composite. Sample 1 (olive circles), as-prepared composite where IBP amorphous coexists with a nanocrystalline fraction; sample 2 (olive triangles), composite previously submitted to thermal treatment at $80^{\circ} \mathrm{C}$ in order to melt the confined nanocrystals. Mean \pm standard deviation, $n=6$. Black squares are for IBP crystal dissolution. 
matrices ${ }^{24,58-60}$ and for other drug-MPS composites ${ }^{61-64}$. The initial burst could be attributed to the fast dissolution and release of the portion of ibuprofen located at the extremities of the channels while the sustained release is caused by the hindranced penetration of the release medium deeply inside the channels due to the constrained geometry ${ }^{24}$.

It is noticeable that a small amount of crystallized matter $(\sim 8 \%)$ has a detectable effect on the sustained release of IBP while maintaining the high solubility rate compared to the bulk crystal form I. Figure 9 shows that the release of IBP is longer in presence of small fraction of nanocrystallized matter compared to the fully amorphous state under confinement, as it can be expected for a poorly water-soluble material. These results show that manipulating the physical state of the confined API is a way to sustain the release time ( +20 minutes with nanocrystals to reach $95 \%$ of drug released) as well as the amount of drug released during the burst effect period (+15\% with melted nanocrystals). This effect can be combined with the influence of the pore size $\mathrm{s}^{24,65}$ and the surface chemistry $24,66,67$ in a more complex formulation in order to have a better control of the release profile.

\section{Interpretation of physical state distribution of IBP within the}

\section{IBP35\%:SBA-15[9nm] composite}

All the results obtained from TGA experiments, from the analysis of the glass transition and from Raman mapping allow to determine the main parameters responsible for the nature of the physical state of IBP confined in SBA-15[9nm] matrix by using the MAL method. Two types of parameters must be considered for understanding the great heterogeneity of physical states observed in the Raman image 
(figures 7 and 8), namely (i) parameters related to the pore architecture and (ii) parameters intrinsic to the API molecule.

(i) The distribution of pore size and the chemical surface of the SBA15[9nm] matrix have an important influence on the physical state of confined IBP combined with

(ii) The capabilities of the IBP molecule to bind with silanol groups via $\mathrm{H}$ bonding, and the molecular packing distinctive of the crystalline form I identified within pores.

Obviously, the similarity between results about the glass transition obtained from investigations carried out on pores loaded by impregnation and by MAL (present study), indicates that all channels are equivalently filled by IBP molecules.

Consequently, the different number of molecules from one channel to another is likely resulting from the different pore size. Additionally, it can be assumed that the MAL method makes possible the loading of nuclei inside the pores. In this context, the nature of the physical state (amorphous or crystalline) of confined IBP depends on the ability to arrange the number of dimers needed for the crystal growth of form I. The size effect, very clearly highlighted in the Raman image, can be explained from the consideration that silanol groups are not periodically distributed at the pore surface and IBP can be bound to silanol groups via two finely different $\mathrm{H}$-bonding associations ${ }^{22}$ inherent to the silanol group distribution. Unfortunately, the physical state in the micropores, which are a parameter specific to SBA matrix, cannot be probed and considered in the discussion. The existence of two types of binding to the pore surface as well as the roughness of the surface are sources of defects in ordering of dimers, inducing a significant heterogeneity in the crystallite size from the nano to micrometer scale. 


\section{Conclusion}

In the present study, the contribution of MAL method for manipulating confined matter was analyzed. A special attention was given to the influence of the loading degree on the physical state of confined ibuprofen within SBA-15 with an average pore diameter of $9.4 \mathrm{~nm}$. The combination of several complementary investigations (TGA, DSC, XRPD, $\mu$-Raman spectroscopy) has allowed us to propose a physical state scheme of the confined ibuprofen in this SBA-15 matrix.

It was shown that using the MAL method allows to manipulate the physical state of confined ibuprofen, depending on the loading degree. A low crystalline degree of ibuprofen can be detected in the 35\%-loaded composite, while the composite is fully amorphous in the $25 \%$-loaded composite. This suggests a density-driven mechanism of crystallization under confinement. It was shown that the lowering of the melting temperature of confined crystallites is induced by the diameter of pores, and not by the size of crystallites (determined by XRPD) along the channel direction, which are much larger than the pores diameter. DSC experiments showed that these crystals are located in the core of the channel coexisting with amorphous IBP and surrounded by an amorphous layer interacting with the surface, the thickness of which was estimated to be approximatively one ibuprofen molecule $(\sim 1 \mathrm{~nm})$. Moreover, a careful investigation of the glass transition from DSC experiments, and the description of the confined physical state by Raman mapping have allowed us to determine the relevant parameters for understanding the complex nature of the physical state of confined IBP loaded by the MAL method. The Raman mapping has clearly revealed heterogeneities in the physical state via a variety of band-shapes (more or less structured) of the lowwavenumber spectrum. These results can be interpreted by inhomogeneous crystallite 
size along the channel length and/or different physical states between neighboring pores in the core of the channels.

This coexistence of amorphous and crystalline states confined into the SBA-15 used in this study was obtained only with the solid-state loading (MAL method).

Drug release analyzes have shown that the confinement of IBP leads to a very high solubility and rapid release from the SBA-15[9nm] matrices for amorphous IBP. The release of IBP can be prolonged by slightly manipulating the physical state, e.g. by initiating a poor crystallization, while maintaining the high solubility of the amorphous state.

\section{Supplementary material}

See supplementary material for details of nitrogen adsorption-desorption experiments, filling degree calculations, ibuprofen solubility calibration, replication of DSC experiments on IBP35\%:SBA-15[9nm] composites, DSC experiments on IBP35\%:SBA-15[9nm] loaded by liquid imbibition, ibuprofen melting temperature depression from several porous materials, angular dependence of the FWHM of ibuprofen crystals from XRPD experiments.

\section{Acknowledgements}

Authors deeply acknowledge Dr. Patrick Derollez (UMET, Univ Lille) for the XRay data analysis and useful discussions, Florence Danède (UMET, Univ Lille) and Laurent Paccou (UMET, Univ Lille) for their help in X-Ray diffraction, thermogravimetric, and Raman experiments. 
The data that support the findings of these studies are available from the corresponding author upon reasonable request. 


\section{References}

${ }^{1}$ N.J. Babu and A. Nangia, Cryst. Growth Des. 11, 2662 (2011).

${ }^{2}$ N. Shah, H. Sandhu, D.S. Choi, H. Chokshi, and A.W. Malick, editors, Amorphous Solid Dispersions (Springer New York, New York, NY, 2014).

${ }^{3}$ M. Vallet-Regí, M. Colilla, I. Izquierdo-Barba, and M. Manzano, Molecules 23, 1 (2018).

${ }^{4}$ J. Vallet-Regi, M., Ramila, A., Del Real, R. P., \& Perez-Pariente, Chem. Mater. 13, 308 (2001).

${ }^{5}$ E. Juère and F. Kleitz, Microporous Mesoporous Mater. 270, 109 (2018).

${ }^{6}$ M. Beiner, G.T. Rengarajan, S. Pankaj, D. Enke, and M. Steinhart, Nano Lett. 7, 1381 (2007).

${ }^{7}$ G.T. Rengarajan, D. Enke, M. Steinhart, and M. Beiner, J. Mater. Chem. 18, 2537 (2008).

${ }^{8}$ C. Alba-Simionesco, B. Coasne, G. Dosseh, G. Dudziak, K.E. Gubbins, R. Radhakrishnan, and M. Sliwinska-Bartkowiak, J. Phys. Condens. Matter 18, R15 (2006).

${ }_{9}^{9}$ M. Alcoutlabi and G.B. McKenna, J. Phys. Condens. Matter 17, R461 (2005).

${ }^{10}$ C.L. Jackson and G.B. McKenna, Chem. Mater. 8, 2128 (1996).

11 S. Cheng and G.B. McKenna, Mol. Pharm. 16, 856 (2019).

${ }^{12}$ M. Sliwinska-Bartkowiak, G. Dudziak, R. Sikorski, R. Gras, R. Radhakrishnan, and K.E. Gubbins, J. Chem. Phys. 114, 950 (2001).

${ }^{13}$ G. Dosseh, Y. Xia, and C. Alba-Simionesco, J. Phys. Chem. B 107, 6445 
(2003).

${ }^{14}$ S. Jähnert, F. Vaca Chávez, G.E. Schaumann, A. Schreiber, M. Schönhoff, and G.H. Findenegg, Phys. Chem. Chem. Phys. 10, 6039 (2008).

15 J.S. D’Arrigo, Am. J. Physiol. Physiol. 235, C109 (1978).

16 D. Wallacher and K. Knorr, Phys. Rev. B 63, 104202 (2001).

17 O. Petrov and I. Furó, Phys. Rev. E - Stat. Nonlinear, Soft Matter Phys. 73, 1 (2006).

18 N. Sonnenberger, N. Anders, Y. Golitsyn, M. Steinhart, D. Enke, K. Saalwächter, and M. Beiner, Chem. Commun. 52, 4466 (2016).

19 G.H. Findenegg, S. Jähnert, D. Akcakayiran, and A. Schreiber, ChemPhysChem 9, 2651 (2008).

20 J. Riikonen, E. Mäkilä, J. Salonen, and V.-P. Lehto, Langmuir 25, 6137 (2009).

${ }^{21}$ K.P. Nartowski, D. Malhotra, L.E. Hawarden, J. Sibik, D. luga, J.A. Zeitler, L. Fábián, and Y.Z. Khimyak, Angew. Chemie Int. Ed. 55, 8904 (2016).

${ }^{22}$ A.R. Brás, I.M. Fonseca, M. Dionísio, A. Schönhals, F. Affouard, and N.T. Correia, J. Phys. Chem. C 118, 13857 (2014).

23 R. Mellaerts, J.A.G. Jammaer, M. Van Speybroeck, H. Chen, J. Van Humbeeck, P. Augustijns, G. Van den Mooter, and J.A. Martens, Langmuir 24, 8651 (2008).

${ }^{24}$ I. Izquierdo-Barba, E. Sousa, J.C. Doadrio, A.L. Doadrio, J.P. Pariente, A. Martínez, F. Babonneau, and M. Vallet-Regí, J. Sol-Gel Sci. Technol. 50, 421 (2009).

${ }^{25}$ A.R. Brás, E.G. Merino, P.D. Neves, I.M. Fonseca, M. Dionísio, A. Schönhals, 
and N.T. Correia, J. Phys. Chem. C 115, 4616 (2011).

${ }^{26}$ B. Malfait, N.T. Correia, A. Mussi, L. Paccou, Y. Guinet, and A. Hédoux, Microporous Mesoporous Mater. 277, 203 (2019).

${ }^{27}$ C. Charnay, S. Bégu, C. Tourné-Péteilh, L. Nicole, D.A. Lerner, and J.M. Devoisselle, Eur. J. Pharm. Biopharm. 57, 533 (2004).

${ }^{28}$ E. Skorupska, A. Jeziorna, and M.J. Potrzebowski, J. Phys. Chem. C 120, 13169 (2016).

${ }^{29}$ D. Zhao, J. Feng, Q. Huo, N. Melosh, G.H. Fredrickson, B.F. Chmelka, and G.D. Stucky, Science. 279, 548 (1998).

${ }^{30}$ E.P. Barrett, L.G. Joyner, and P.P. Halenda, J. Am. Chem. Soc. 73, 373 (1951).

${ }^{31}$ S. Brunauer, P.H. Emmett, and E. Teller, J. Am. Chem. Soc. 60, 309 (1938).

${ }^{32}$ M. Evain, P. Deniard, A. Jouanneaux, and R. Brec, J. Appl. Crystallogr. 26, 563 (1993).

${ }^{33}$ A. Le Bail, H. Duroy, and J.L. Fourquet, Mater. Res. Bull. 23, 447 (1988).

${ }^{34}$ Rodriguez-Carvajal, J. FullProf Version 4.80. ILL, Grenoble, France 2010.

${ }^{35}$ P. Thompson, D.E. Cox, and J.B. Hastings, J. Appl. Crystallogr. 20, 79 (1987).

${ }^{36}$ A. Hédoux, Adv. Drug Deliv. Rev. 100, 133 (2016).

${ }^{37}$ M. Gibaldi and S. Feldman, J. Pharm. Sci. 56, 1238 (1967).

${ }^{38}$ S. Dash, P.N. Murthy, L. Nath, and P. Chowdhury, Acta Pol. Pharm. Res. 67, 217 (2010).

${ }^{39}$ K.D. Rainsford, Ibuprofen: Discovery, Development and Therapeutics (2015). 
${ }^{40}$ L.R. Shaw, W.J. Irwin, T.J. Grattan, and B.R. Conway, Drug Dev. Ind. Pharm. 31,515 (2005).

${ }^{41}$ E. Dudognon, F. Danède, M. Descamps, and N.T. Correia, Pharm. Res. 25, 2853 (2008).

42 S. Lerdkanchanaporn and D. Dollimore, J. Therm. Anal. 49, 879 (1997).

${ }^{43}$ K.K. Qian, W. Zhou, X. Xu, and T.J. Udovic, Pharm. Res. 29, 2432 (2012).

${ }^{44}$ E.G. Merino, P.D. Neves, I.M. Fonseca, F. Danéde, A. Idrissi, C.J. Dias, M. Dionísio, and N.T. Correia, J. Phys. Chem. C 117, 21516 (2013).

45 C. Le Quellec, G. Dosseh, F. Audonnet, N. Brodie-Linder, C. AlbaSimionesco, W. Häussler, and B. Frick, Eur. Phys. J. Spec. Top. 141, 11 (2007).

${ }^{46}$ A. Minecka, E. Kaminska, M. Tarnacka, A. Talik, I. Grudzka-Flak, K. Wolnica, M. Dulski, K. Kaminski, and M. Paluch, Phys. Chem. Chem. Phys. 20, 30200 (2018).

47 W.H. Thompson, J. Chem. Phys. 149, 170901 (2018).

48 J.-Y. Park and G.B. McKenna, Phys. Rev. B 61, 6667 (2000).

${ }^{49}$ M. Vallet-Regi, A. Rámila, R.P. Del Real, and J. Pérez-Pariente, Chem. Mater. 13, 308 (2001)

${ }^{50}$ N. Koga, in Handb. Therm. Anal. Calorimetry, Recent Adv. Tech. Appl., edited by S. Vyazovkin, N. Koga, and C. Schick, Second edi (Elsevier, 2018), p. 222.

${ }^{51}$ M. Dionísio, N.T. Correia, and A.R. Brás, in Dyn. Geom. Confin. (2014), pp. $213-245$.

52 S.C. Shen, W.K. Ng, L. Chia, J. Hu, and R.B.H. Tan, Int. J. Pharm. 410, 188 (2011).

${ }^{53}$ C.L. Jackson and G.B. McKenna, J. Chem. Phys. 93, 9002 (1990). 
${ }^{54}$ S. Shimizu, K.V. Agrawal, M. O’Mahony, L.W. Drahushuk, N. Manohar, A.S. Myerson, and M.S. Strano, Langmuir 31, 10113 (2015).

${ }^{55}$ M. O’Mahony, A.K. Leung, S. Ferguson, B.L. Trout, and A.S. Myerson, Org. Process Res. Dev. 19, 1109 (2015).

${ }^{56}$ F. Rehman, A. Rahim, C. Airoldi, and P.L.O. Volpe, Mater. Sci. Eng. C 59, 970 (2016).

57 J. Andersson, J. Rosenholm, S. Areva, and M. Lindén, Chem. Mater. 16, 4160 (2004).

${ }^{58}$ M. Van Speybroeck, V. Barillaro, T. Do Thi, R. Mellaerts, J. Martens, J. Van Humbeeck, J. Vermant, P. Annaert, G. Van Den Mooter, and P. Augustijns, J. Pharm. Sci. 98, 2648 (2009).

${ }^{59}$ M.M. Wan, Y.Y. Li, T. Yang, T. Zhang, X.D. Sun, and J.H. Zhu, Chem. - A Eur. J. 22, 6294 (2016).

60 S.C. Shen, W.K. Ng, J. Hu, K. Letchmanan, J. Ng, and R.B.H. Tan, Adv. Powder Technol. 28, 1316 (2017).

${ }^{61}$ V. Ambrogi, L. Perioli, F. Marmottini, O. Accorsi, C. Pagano, M. Ricci, and C. Rossi, Microporous Mesoporous Mater. 113, 445 (2008).

${ }^{62}$ F. Balas, M. Manzano, P. Horcajada, and M. Vallet-Regi, J. Am. Chem. Soc. 128, 8116 (2006).

${ }^{63}$ V. Morales, A. Martín, J. Ortiz-Bustos, R. Sanz, and R.A. García-Muñoz, J. Mater. Sci. 54, 11635 (2019).

64 J. Ortiz-Bustos, A. Martín, V. Morales, R. Sanz, and R.A. García-Muñoz, Microporous Mesoporous Mater. 240, 236 (2017). 
${ }^{65}$ P. Horcajada, A. Rámila, J. Pérez-Pariente, and M. Vallet-Regí, Microporous Mesoporous Mater. 68, 105 (2004).

${ }^{66}$ A. Baeza, M. Colilla, and M. Vallet-Regí, Expert Opin. Drug Deliv. 12, 319 (2015).

67 J.C. Doadrio, E.M.B. Sousa, I. Izquierdo-Barba, A.L. Doadrio, J. PerezPariente, and M. Vallet-Regí, J. Mater. Chem. 16, 462 (2006). 


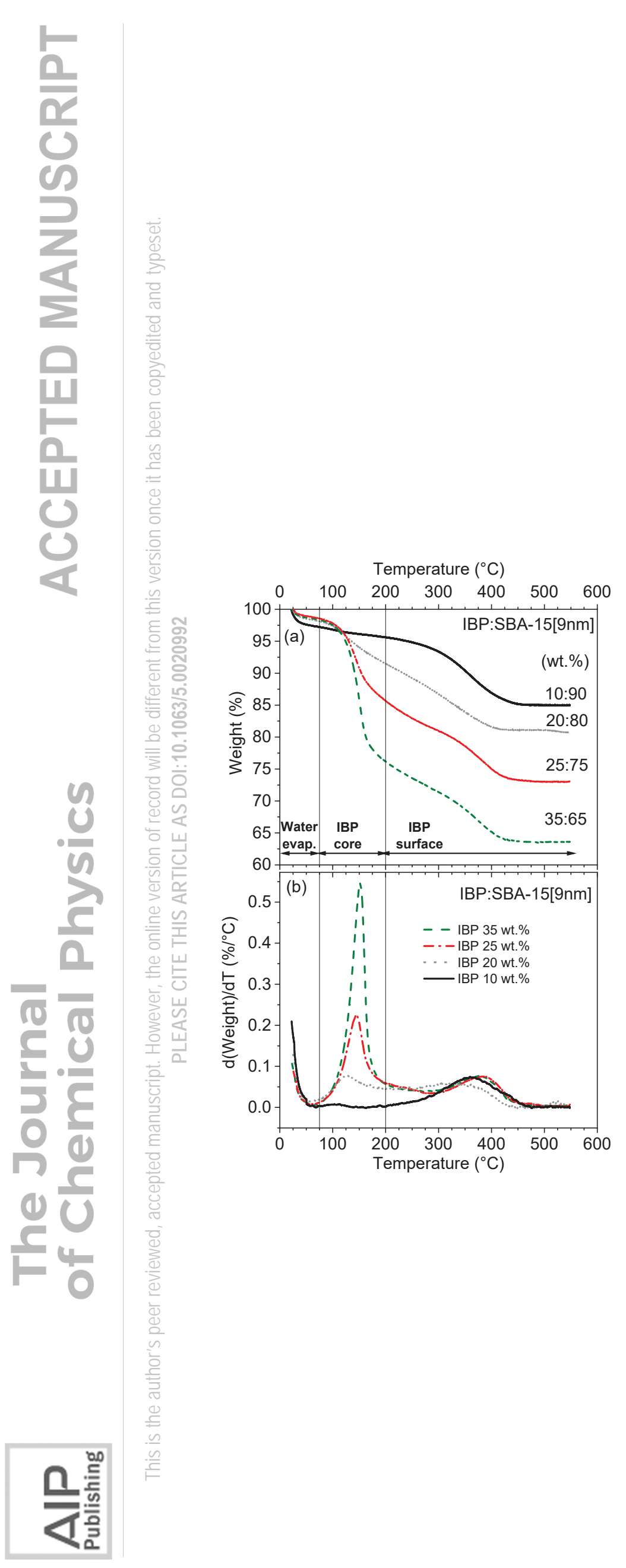




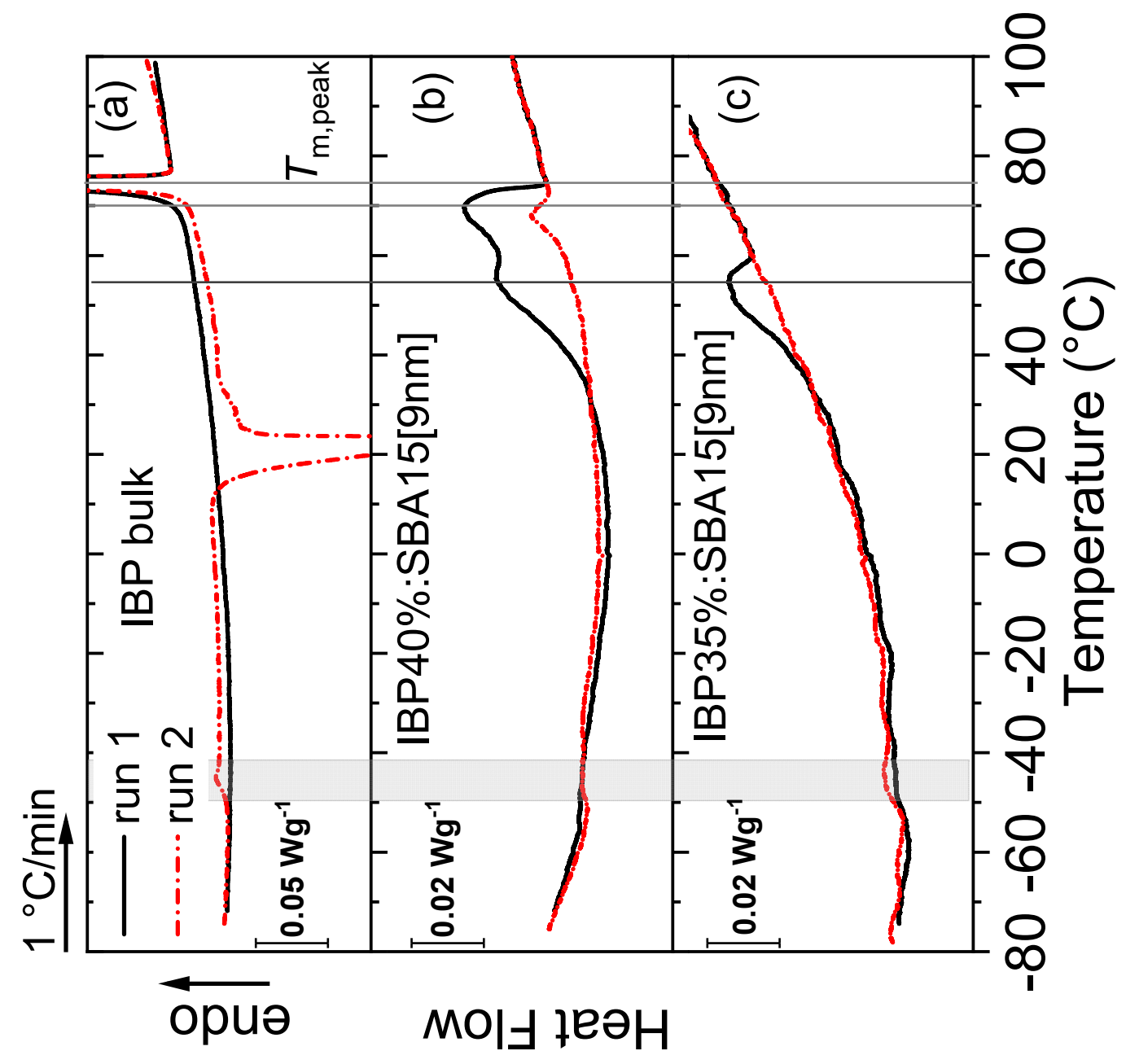




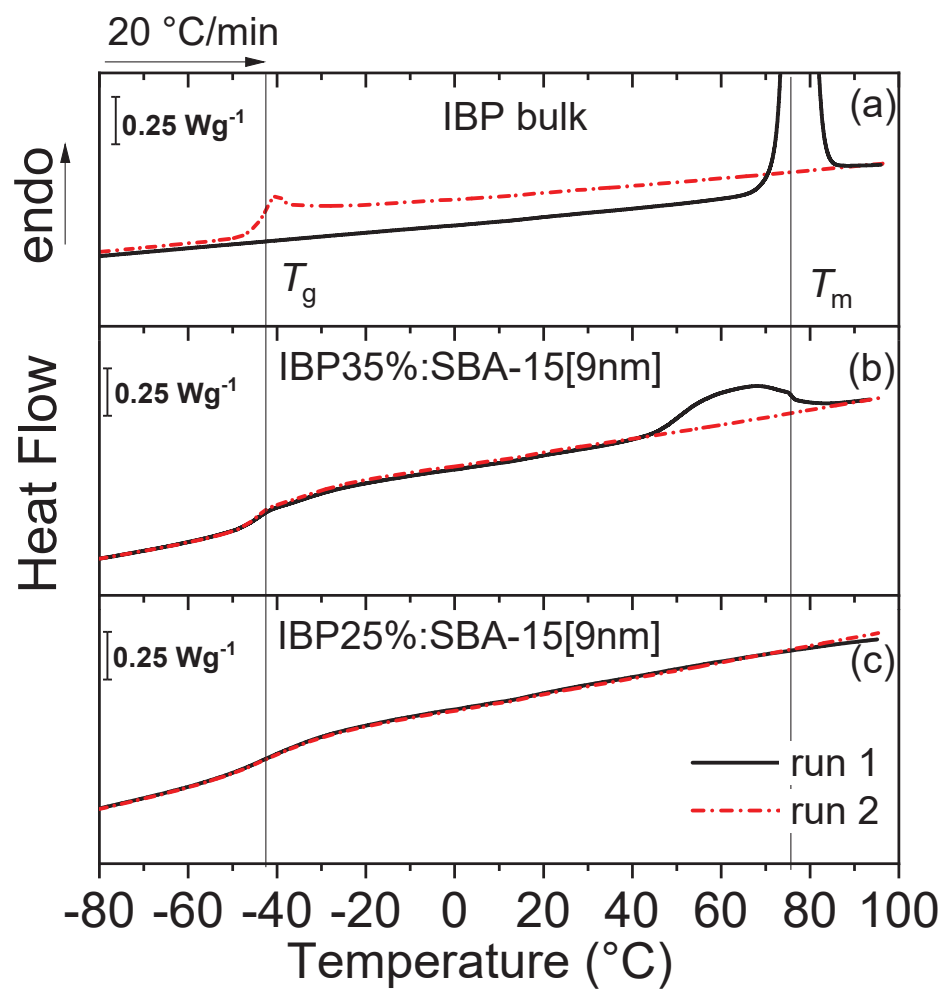




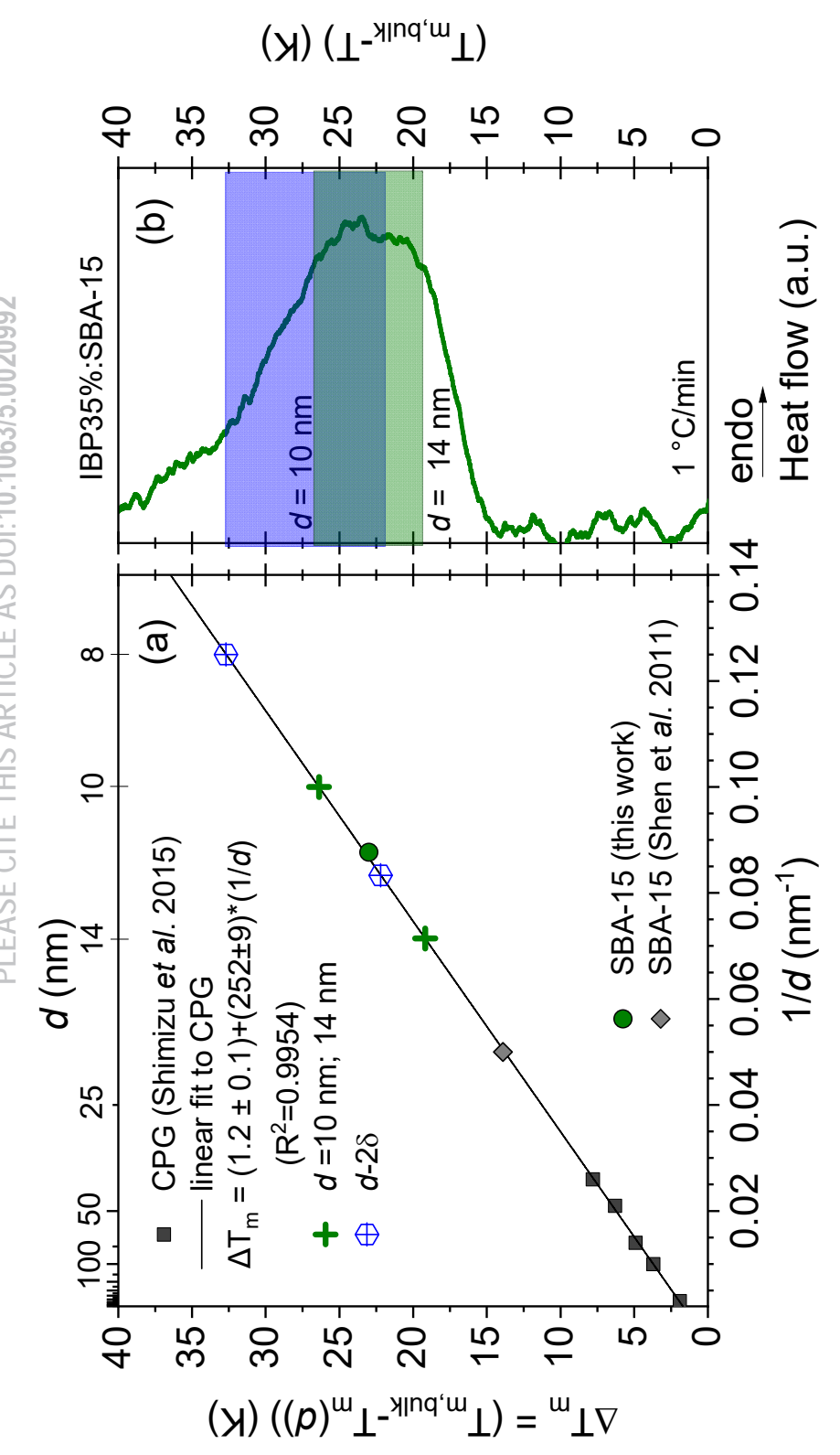




\section{$2 \theta\left({ }^{\circ}\right)$}

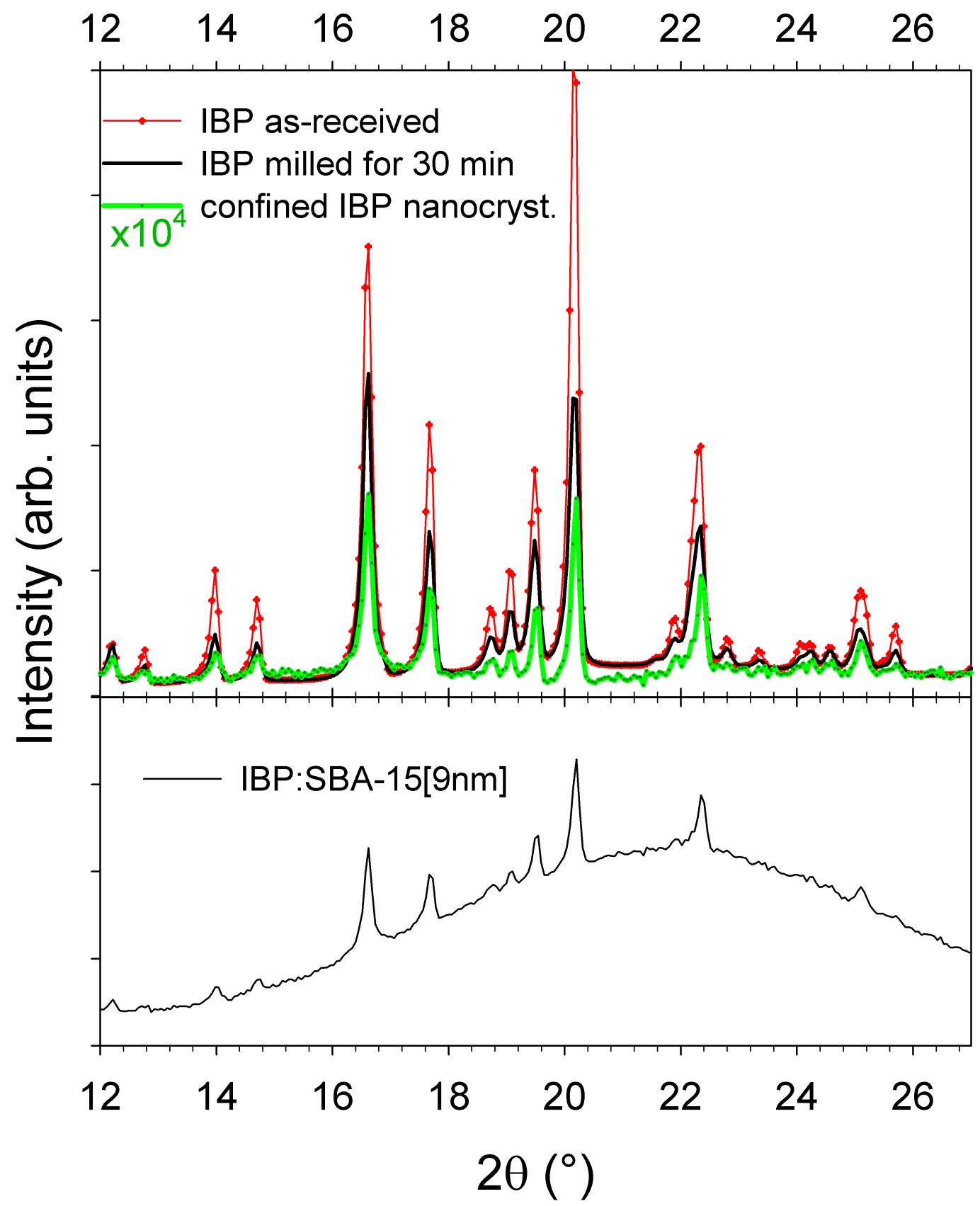



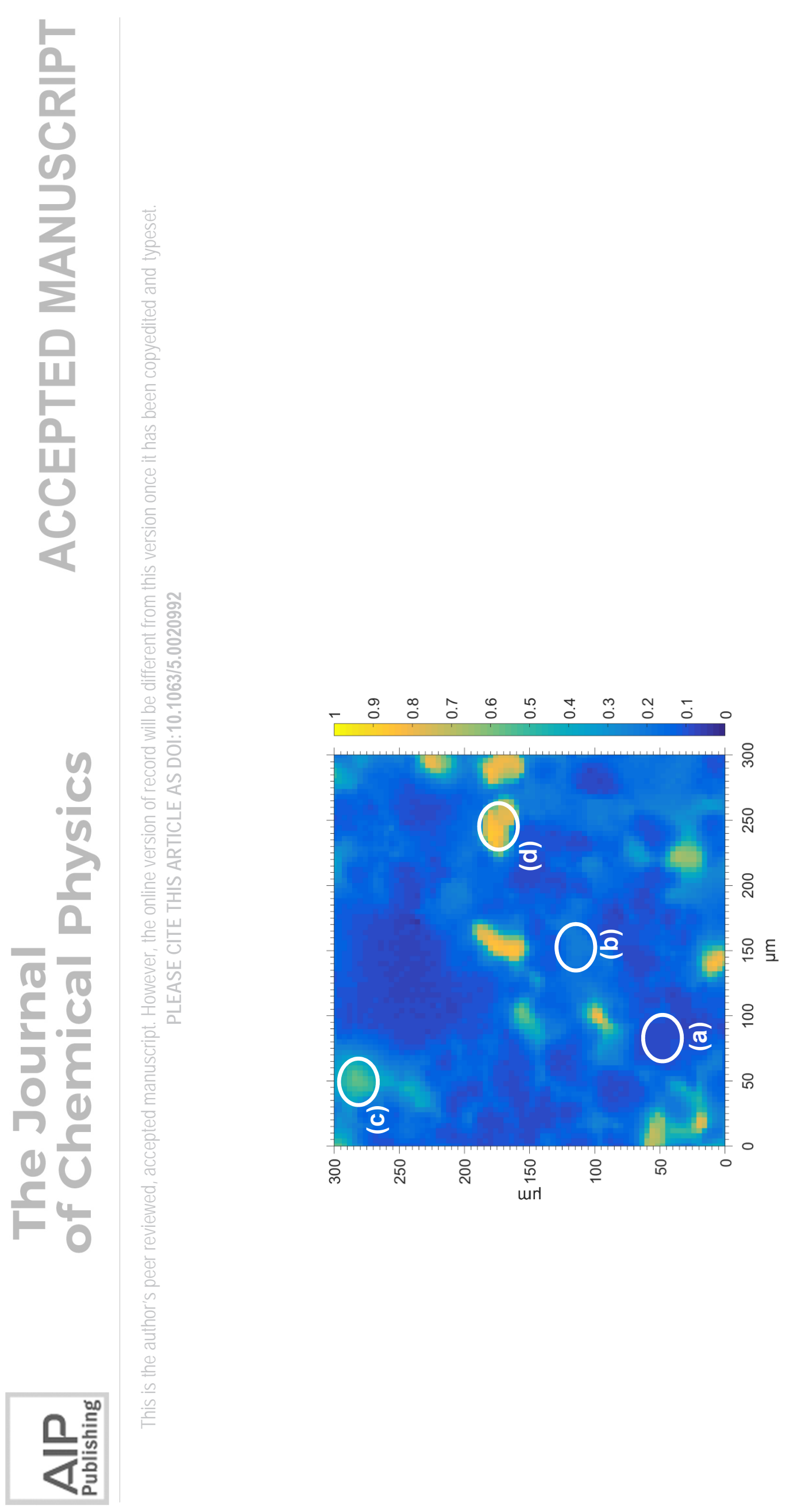


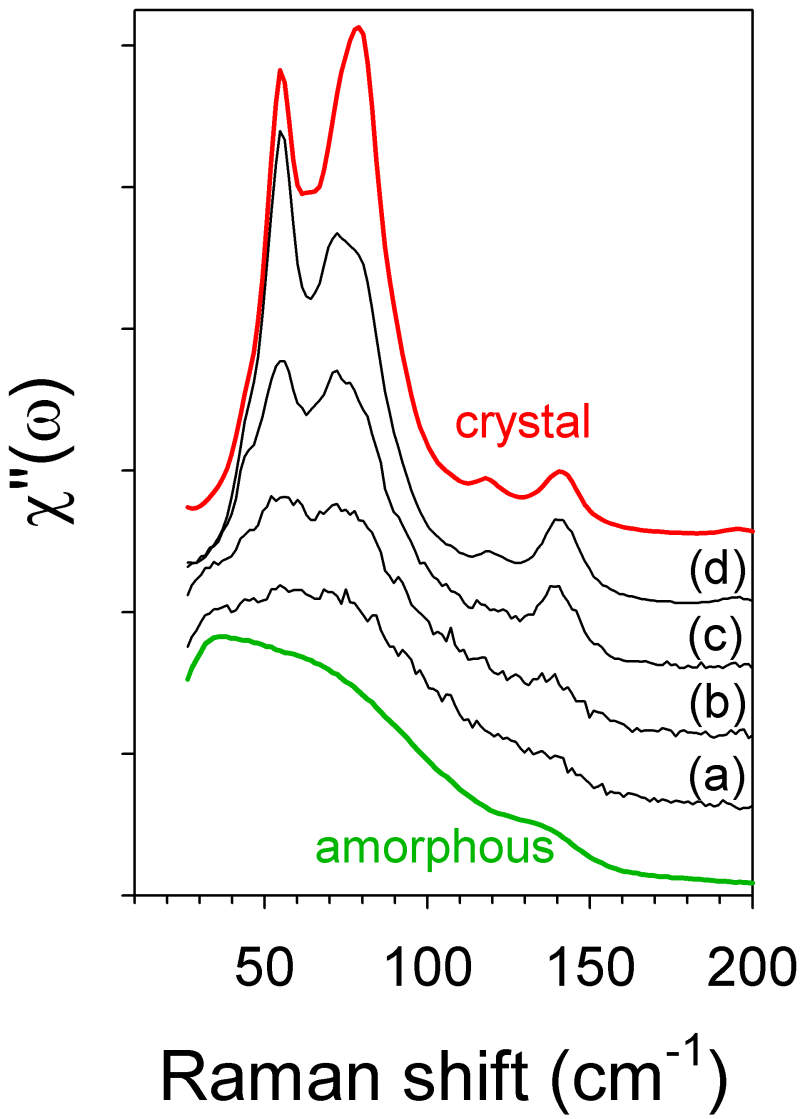




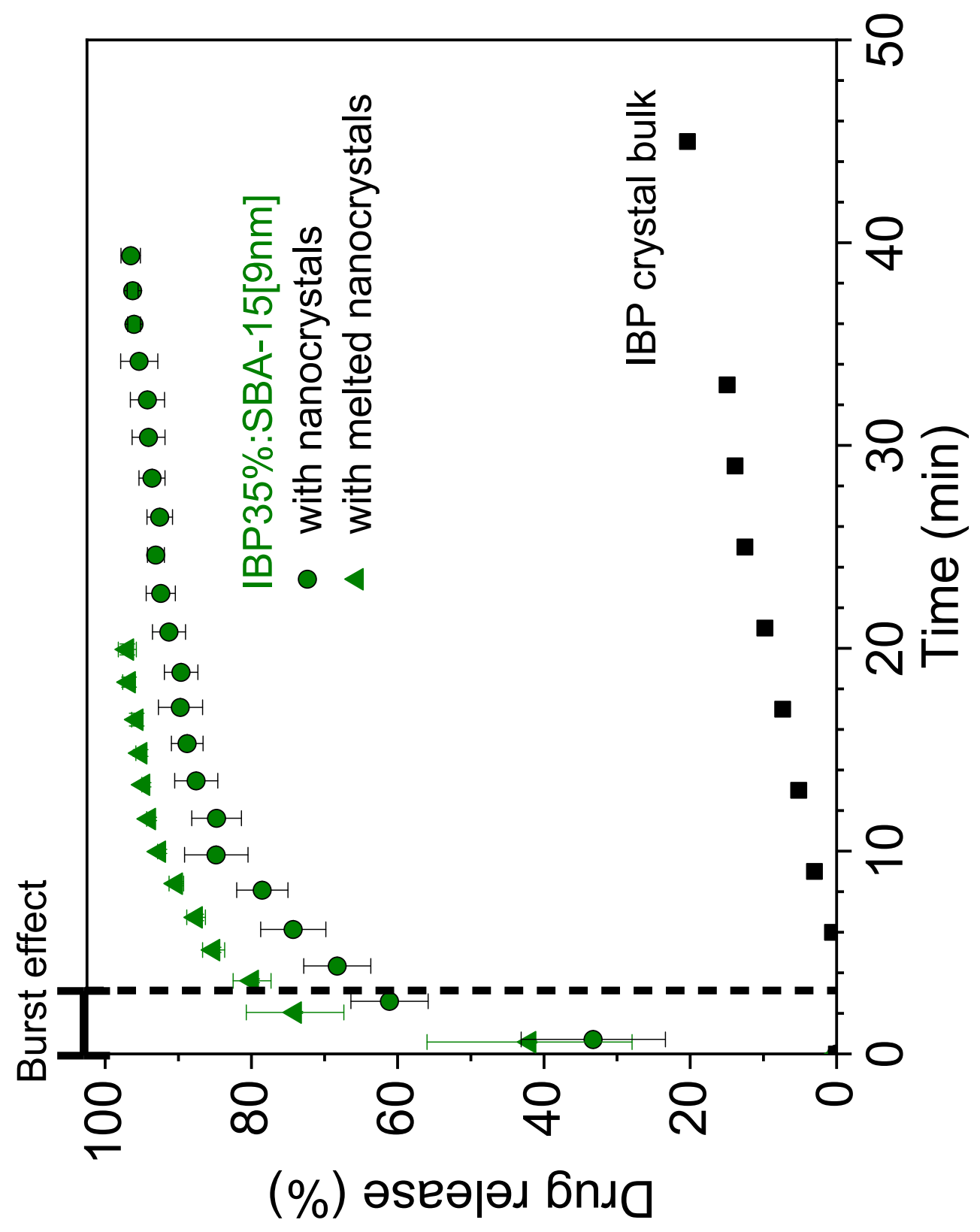

